\title{
Uniform and weak stability of Bresse system with two infinite memories
}

Aissa Guesmia and Mokhtar Kirane

\begin{abstract}
In this paper, we consider one-dimensional linear Bresse systems in a bounded open domain under DirichletNeumann-Neumann boundary conditions with two infinite memories acting only on two equations. First, we establish the well-posedness in the sense of semigroup theory. Then, we prove two (uniform and weak) decay estimates depending on the speeds of wave propagations, the smoothness of initial data and the arbitrarily growth at infinity of the two relaxation functions.
\end{abstract}

Mathametics Subject Classification. 35B40, 35L45, 74H40, 93D20, 93D15.

Keywords. Bresse system, Infinite memory, Well-posedness, Asymptotic behavior, Semigroup theory, Energy method.

\section{Introduction}

In this paper, we consider the Bresse system in one-dimensional open bounded domain under the homogeneous Dirichlet-Neumann-Neumann boundary conditions and with two infinite memories acting on the second and third equations

$$
\left\{\begin{array}{l}
\rho_{1} \varphi_{t t}-k_{1}\left(\varphi_{x}+\psi+l w\right)_{x}-l k_{3}\left(w_{x}-l \varphi\right)=0, \\
\rho_{2} \psi_{t t}-k_{2} \psi_{x x}+k_{1}\left(\varphi_{x}+\psi+l w\right)+\int_{0}^{+\infty} g_{2}(s) \psi_{x x}(x, t-s) \mathrm{d} s=0, \\
\rho_{1} w_{t t}-k_{3}\left(w_{x}-l \varphi\right)_{x}+l k_{1}\left(\varphi_{x}+\psi+l w\right)+\int_{0}^{+\infty} g_{3}(s) w_{x x}(x, t-s) \mathrm{d} s=0, \\
\varphi(0, t)=\psi_{x}(0, t)=w_{x}(0, t)=\varphi(L, t)=\psi_{x}(L, t)=w_{x}(L, t)=0, \\
\varphi(x, 0)=\varphi_{0}(x), \varphi_{t}(x, 0)=\varphi_{1}(x), \\
\psi(x,-t)=\psi_{0}(x, t), \psi_{t}(x, 0)=\psi_{1}(x), \\
w(x,-t)=w_{0}(x, t), w_{t}(x, 0)=w_{1}(x)
\end{array}\right.
$$


or on the first and third equations

$$
\left\{\begin{array}{l}
\rho_{1} \varphi_{t t}-k_{1}\left(\varphi_{x}+\psi+l w\right)_{x}-l k_{3}\left(w_{x}-l \varphi\right)+\int_{0}^{+\infty} g_{1}(s) \varphi_{x x}(x, t-s) \mathrm{d} s=0, \\
\rho_{2} \psi_{t t}-k_{2} \psi_{x x}+k_{1}\left(\varphi_{x}+\psi+l w\right)=0, \\
\rho_{1} w_{t t}-k_{3}\left(w_{x}-l \varphi\right)_{x}+l k_{1}\left(\varphi_{x}+\psi+l w\right)+\int_{0}^{+\infty} g_{3}(s) w_{x x}(x, t-s) \mathrm{d} s=0, \\
\varphi(0, t)=\psi_{x}(0, t)=w_{x}(0, t)=\varphi(L, t)=\psi_{x}(L, t)=w_{x}(L, t)=0, \\
\varphi(x,-t)=\varphi_{0}(x, t), \varphi_{t}(x, 0)=\varphi_{1}(x), \\
\psi(x, 0)=\psi_{0}(x), \psi_{t}(x, 0)=\psi_{1}(x), \\
w(x,-t)=w_{0}(x, t), w_{t}(x, 0)=w_{1}(x)
\end{array}\right.
$$

or on the first two equations

$$
\left\{\begin{array}{l}
\rho_{1} \varphi_{t t}-k_{1}\left(\varphi_{x}+\psi+l w\right)_{x}-l k_{3}\left(w_{x}-l \varphi\right)+\int_{0}^{+\infty} g_{1}(s) \varphi_{x x}(x, t-s) \mathrm{d} s=0, \\
\rho_{2} \psi_{t t}-k_{2} \psi_{x x}+k_{1}\left(\varphi_{x}+\psi+l w\right)+\int_{0}^{+\infty} g_{2}(s) \psi_{x x}(x, t-s) \mathrm{d} s=0, \\
\rho_{1} w_{t t}-k_{3}\left(w_{x}-l \varphi\right)_{x}+l k_{1}\left(\varphi_{x}+\psi+l w\right)=0, \\
\varphi(0, t)=\psi_{x}(0, t)=w_{x}(0, t)=\varphi(L, t)=\psi_{x}(L, t)=w_{x}(L, t)=0, \\
\varphi(x,-t)=\varphi_{0}(x, t), \varphi_{t}(x, 0)=\varphi_{1}(x), \\
\psi(x,-t)=\psi_{0}(x, t), \psi_{t}(x, 0)=\psi_{1}(x), \\
w(x, 0)=w_{0}(x), w_{t}(x, 0)=w_{1}(x),
\end{array}\right.
$$

where $(x, t) \in] 0, L\left[\times \mathbb{R}_{+}, g_{i}: \mathbb{R}_{+} \rightarrow \mathbb{R}_{+}\right.$are given functions, and $L, l, \rho_{i}, k_{i}$ are positive constants. The infinite integrals in systems (1)-(3) represent the infinite memories, and the state (unknown) is

$$
(\varphi, \psi, w):] 0, L[\times] 0,+\infty\left[\rightarrow \mathbb{R}^{3} .\right.
$$

The derivative of a generic function $f$ with respect to a variable $y$ is noted $f_{y}$ or $\partial_{y} f$. If $f$ has only one variable, its derivative is noted $f^{\prime}$. For simplicity of notation, the space $x$ and/or the time $t$ and $s$ variables are used only when it is necessary to avoid ambiguity.

Our goal is to study the well-posedness and the asymptotic stability of these systems in terms of the growth at infinity of $g_{i}$, the smoothness of initial data $\left(\varphi_{0}, \psi_{0}, w_{0}, \varphi_{1}, \psi_{1}, w_{1}\right)$ and the speeds of wave propagations defined by

$$
s_{1}=\sqrt{\frac{k_{1}}{\rho_{1}}}, \quad s_{2}=\sqrt{\frac{k_{2}}{\rho_{2}}} \text { and } s_{3}=\sqrt{\frac{k_{3}}{\rho_{1}}} .
$$

The Bresse system is known as the circular arch problem and is given by the following equations:

$$
\left\{\begin{array}{l}
\rho_{1} \varphi_{t t}=Q_{x}+l N+F_{1}, \\
\rho_{2} \psi_{t t}=M_{x}-Q+F_{2}, \\
\rho_{1} w_{t t}=N_{x}-l Q+F_{3},
\end{array}\right.
$$


where

$$
N=k_{0}\left(w_{x}-l \varphi\right), \quad Q=k\left(\varphi_{x}+l w+\psi\right) \quad \text { and } \quad M=b \psi_{x},
$$

$\rho_{1}, \rho_{2}, l, k, k_{0}$ and $b$ are positive constants, $N, Q$ and $M$ denote, respectively, the axial force, the shear force and the bending moment, and $w, \varphi$ and $\psi$ represent, respectively, the longitudinal, vertical and shear angle displacements. Here

$$
\rho_{1}=\rho A, \quad \rho_{2}=\rho I, \quad k_{0}=E A, \quad k=k^{\prime} G A, \quad b=E I \quad \text { and } \quad l=R^{-1},
$$

where $\rho, E, G, k^{\prime}, A, I$ and $R$ are positive constants and denote, respectively, the density, the modulus of elasticity, the shear modulus, the shear factor, the cross-sectional area, the second moment of area of the cross section and the radius of curvature. Finally, by $F_{i}$ we are denoting external forces in $] 0, L[\times] 0,+\infty[$ together with initial and boundary conditions. For more details, we refer to Lagnese et al. [14] and [15].

If we consider

$$
\left(F_{1}, F_{2}, F_{3}\right)=\left(0,-\gamma \psi_{t}, 0\right)
$$

with $\gamma>0$, we obtain the system considered by Bresse [3] consisting of three coupled wave equations.

The most important asymptotic behavior result of the Bresse system is due to Liu and Rao [16] obtained for a thermoelastic Bresse system which consists of the Bresse system with

$$
\left(F_{1}, F_{2}, F_{3}\right)=(0,0,0)
$$

and two heat equations coupled in a certain manner, where the two wave equations about the longitudinal and shear angle displacements are effectively globally damped by the dissipation from the two heat equations. They proved that the norm of solutions in the energy space decays exponentially to zero at infinity if and only if

$$
s_{1}=s_{2}=s_{3} .
$$

Otherwise, the norm of solutions decays polynomially to zero with rates depending on the regularity of the initial data. For the classical solutions, these rates are $t^{-\frac{1}{4}+\epsilon}$ or $t^{-\frac{1}{8}+\epsilon}$ provided that the boundary conditions are of Dirichlet-Neumann-Neumann or Dirichlet-Dirichlet-Dirichlet type, respectively, where $\epsilon$ is an arbitrary positive constant. Very similar results to the ones of [16] are obtained in [8] for the Bresse system (in case (6)) coupled with only one heat equation in a certain manner, where the obtained decay rate for classical solutions when $(7)$ is not satisfied is $t^{-\frac{1}{6}+\epsilon}$ in general and $t^{-\frac{1}{3}+\epsilon}$ when $s_{1} \neq s_{2}$ and $s_{1}=s_{3}$. Najdi and Wehbe [17] extended the results of [8] to the case where the thermal dissipation is locally distributed, and improved the polynomial stability estimate when (7) is not satisfied by getting the decay rate $t^{-\frac{1}{2}}$.

Concerning the stability of Bresse systems with (local or global) frictional dampings, we mention here the most known stability results in the literature. Alabau-Boussouira et al. [1] considered the case (5) and proved that the exponential stability is equivalent to (7). Otherwise, they got the same two decay rates as in [8]. The results of [1] were extended and improved in [18] by considering a locally distributed dissipation (that is, $\gamma$ in (5) is replaced by a nonnegative function $a:] 0, L\left[\rightarrow \mathbb{R}_{+}\right.$which is positive only on some part of $] 0, L[)$; the authors of [18] obtained a better decay rate when (7) does not hold. The exponential stability result of [1] was also proved in [21] when $\gamma=a(x)$ and $a:] 0, L[\rightarrow \mathbb{R}$ has a positive average on $] 0, L[$ such that

$$
\left\|a-\int_{0}^{L} a(x) \mathrm{d} x\right\|_{L^{2}(] 0, L[)}
$$

is small enough. This implies that $a$ is allowed to have some negative values on $] 0, L[$, and in such situation, $a \psi_{t}$ is called indefinite damping. Also, some optimal polynomial decay rates for Bresse systems in case (5) were proved in [7] when (7) does not hold. 
In [23] and [24], the authors studied the stability of Bresse systems damped by two locally frictional dampings

$$
\left(F_{1}, F_{2}, F_{3}\right)=\left(0,-a_{1}(x) \psi_{t},-a_{2}(x) w_{t}\right),
$$

where $\left.a_{i}:\right] 0, L\left[\rightarrow \mathbb{R}_{+}\right.$are nonnegative functions which can vanish on some part of $] 0, L[$. They established that the exponential stability remains valid if and only if $s_{1}=s_{2}$. When $s_{1} \neq s_{2}$, a general decay rate depending on the regularity of the initial data is obtained, where, in case of classical solutions, this rate is $t^{-\frac{1}{2}+\epsilon}$.

When only the first and second equations are controlled by linear frictional dampings; that is,

$$
\left(F_{1}, F_{2}, F_{3}\right)=\left(-\gamma_{1} \varphi_{t},-\gamma_{2} \psi_{t}, 0\right)
$$

with $\gamma_{i}>0$, the equivalence between the exponential stability and the equality $s_{1}=s_{3}$ was proved in [2]. When $s_{1} \neq s_{3}$, the polynomial stability was also showed in [2], where the decay rate depends on the regularity of the initial data. In the particular case of classical solutions, the polynomial decay rate of [2] is $t^{-\frac{1}{2}}$ and it is optimal.

In his $\mathrm{PhD}$ thesis [24], Youssef treated the case where the three equations of the Bresse system are all controlled by (linear or nonlinear) frictional dampings; that is,

$$
\left(F_{1}, F_{2}, F_{3}\right)=\left(-h_{1}\left(\varphi_{t}\right),-h_{2}\left(\psi_{t}\right),-h_{3}\left(w_{t}\right)\right),
$$

where $h_{i}: \mathbb{R} \rightarrow \mathbb{R}$ are given functions having a linear or a polynomial growth at zero and infinity, and obtained, respectively, the exponential and polynomial stability for any weak solution. These results are proved regardless to $s_{i}$. The results of [24] were generalized in [4] and [20] to the case

$$
\left(F_{1}, F_{2}, F_{3}\right)=\left(-a_{1}(x) h_{1}\left(\varphi_{t}\right),-a_{2}(x) h_{2}\left(\psi_{t}\right),-a_{3}(x) h_{3}\left(w_{t}\right)\right),
$$

where the nonnegative functions $a_{i}$ are (all or some of them) effective only on some part of $] 0, L[$, and the functions $h_{i}$ can have a general growth at zero (not necessarily of linear or polynomial type). The case of three frictional dampings was also considered in [22] but in the whole space $\mathbb{R}$ (instead of $] 0, L[$ ), and some polynomial stability estimates were obtained.

Concerning the stability of Bresse systems with memories, there are only a few results. When the three equations are controlled via infinite memories of the form

$$
F_{1}=-\int_{0}^{+\infty} g_{1}(s) \varphi_{x x}(x, t-s) \mathrm{d} s, \quad F_{2}=-\int_{0}^{+\infty} g_{2}(s) \psi_{x x}(x, t-s) \mathrm{d} s
$$

and

$$
F_{3}=-\int_{0}^{+\infty} g_{3}(s) w_{x x}(x, t-s) \mathrm{d} s
$$

where $g_{i}: \mathbb{R}_{+} \rightarrow \mathbb{R}_{+}$are differentiable, nonincreasing and integrable functions on $\mathbb{R}_{+}$, the stability was proved in [12] regardless to $s_{i}$. The obtained decay estimate given in [12] depends only on the growth at infinity of $s \mapsto g_{i}(s)$, which is allowed to have a decay rate at infinity arbitrarily close to $\frac{1}{s}$.

As far as we know, the more recent stability results for Bresse systems with memories are those in [6] under only one infinite memory considered in the second equation

$$
\left(F_{1}, F_{2}, F_{3}\right)=\left(0,-\int_{0}^{+\infty} g(s) \psi_{x x}(x, t-s) \mathrm{d} s, 0\right),
$$

where $g: \mathbb{R}_{+} \rightarrow \mathbb{R}_{+}$is a differentiable, nonincreasing and integrable function on $\mathbb{R}_{+}$satisfying

$$
\exists \alpha_{1}, \alpha_{2}>0:-\alpha_{2} g(s) \leq g^{\prime}(s) \leq-\alpha_{1} g(s), \quad \forall s \in \mathbb{R}_{+} .
$$


In [6], it was proved that the exponentially stability holds if and only if (7) is satisfied. Otherwise, the polynomial stability with a decay rate of type $t^{-\frac{1}{2}}$ and its optimality were shown. The condition (9) implies that

$$
g(0) \mathrm{e}^{-\alpha_{2} s} \leq g(s) \leq g(0) \mathrm{e}^{-\alpha_{1} s}, \quad \forall s \in \mathbb{R}_{+},
$$

which implies that $g$ converges exponentially to zero at infinity.

The proof of the results cited above is based on the spectral theory, the frequency domain method and the multipliers technique.

Our goal is to study the well-posedness and asymptotic stability of systems (1)-(3) in terms of the arbitrary growth at infinity of the kernels $g_{i}$, the smoothness of initial data $\left(\varphi_{0}, \psi_{0}, w_{0}, \varphi_{1}, \psi_{1}, w_{1}\right)$ and the speeds of wave propagations (4). We prove that these systems are well posed and their energy converges to zero when time tends to infinity, and we provide two general decay estimates: a strong decay estimate under some restrictions on $v_{i}$ and a weak decay one in general. The proof is based on the semigroup theory for the well-posedness. For the decay estimates, we use the energy method and some differential and/or integral equalities.

The paper is organized as follows. In Sect. 2, we present our assumptions on the functions $g_{i}$ state and prove the well-posedness of (1)-(3). In Sect. 3, we consider some assumptions on the growth of $g_{i}$ at infinity and state our stability results. Finally, the proof of our uniform and weak decay estimates are given, respectively, in Sects. 4 and 5.

\section{Well-posedness}

In this section, we discuss the well-posedness of (1)-(3) using the semigroup approach. Following the method of [5], we consider these new functionals

$$
\begin{cases}\text { Cases }(2) \text { and }(3): \eta_{1}(x, t, s)=\varphi(x, t)-\varphi(x, t-s) & \text { in }] 0, L\left[\times \mathbb{R}_{+} \times \mathbb{R}_{+},\right. \\ \text {Cases (1) and (3): } \eta_{2}(x, t, s)=\psi(x, t)-\psi(x, t-s) & \text { in }] 0, L\left[\times \mathbb{R}_{+} \times \mathbb{R}_{+},\right. \\ \text {Cases (1) and (2): } \eta_{3}(x, t, s)=w(x, t)-w(x, t-s) & \text { in }] 0, L\left[\times \mathbb{R}_{+} \times \mathbb{R}_{+} .\right.\end{cases}
$$

These functionals satisfy

$$
\begin{cases}\partial_{t} \eta_{1}+\partial_{s} \eta_{1}-\varphi_{t}=0 & \text { in }] 0, L\left[\times \mathbb{R}_{+} \times \mathbb{R}_{+},\right. \\ \partial_{t} \eta_{2}+\partial_{s} \eta_{2}-\psi_{t}=0 & \text { in }] 0, L\left[\times \mathbb{R}_{+} \times \mathbb{R}_{+},\right. \\ \partial_{t} \eta_{3}+\partial_{s} \eta_{3}-w_{t}=0 & \text { in }] 0, L\left[\times \mathbb{R}_{+} \times \mathbb{R}_{+},\right. \\ \eta_{1}(0, t, s)=\eta_{1}(L, t, s)=0 & \text { in } \mathbb{R}_{+} \times \mathbb{R}_{+}, \\ \partial_{x} \eta_{2}(0, t, s)=\partial_{x} \eta_{2}(L, t, s)=0 & \text { in } \mathbb{R}_{+} \times \mathbb{R}_{+}, \\ \partial_{x} \eta_{3}(0, t, s)=\partial_{x} \eta_{3}(L, t, s)=0 & \text { in } \mathbb{R}_{+} \times \mathbb{R}_{+}, \\ \eta_{i}(x, t, 0)=0 & \text { in }] 0, L\left[\times \mathbb{R}_{+} .\right.\end{cases}
$$

Let $\eta_{i}^{0}(x, s)=\eta_{i}(x, 0, s)$,

$$
\left\{\begin{array}{l}
U_{1}^{0}=\left(\varphi_{0}, \psi_{0}, w_{0}, \varphi_{1}, \psi_{1}, w_{1}, \eta_{2}^{0}, \eta_{3}^{0}\right)^{T} \\
U_{2}^{0}=\left(\varphi_{0}, \psi_{0}, w_{0}, \varphi_{1}, \psi_{1}, w_{1}, \eta_{1}^{0}, \eta_{3}^{0}\right)^{T} \\
U_{3}^{0}=\left(\varphi_{0}, \psi_{0}, w_{0}, \varphi_{1}, \psi_{1}, w_{1}, \eta_{1}^{0}, \eta_{2}^{0}\right)^{T}
\end{array}\right.
$$


and

$$
\left\{\begin{array}{l}
U_{1}=\left(\varphi, \psi, w, \varphi_{t}, \psi_{t}, w_{t}, \eta_{2}, \eta_{3}\right)^{T}, \\
U_{2}=\left(\varphi, \psi, w, \varphi_{t}, \psi_{t}, w_{t}, \eta_{1}, \eta_{3}\right)^{T} \\
U_{3}=\left(\varphi, \psi, w, \varphi_{t}, \psi_{t}, w_{t}, \eta_{1}, \eta_{2}\right)^{T}
\end{array}\right.
$$

Then, the system $(i), i=1,2,3$, is equivalent to the following abstract one:

$$
\left\{\begin{array}{l}
\partial_{t} U_{i}=\mathcal{A}_{i} U_{i}, \\
U_{i}(t=0)=U_{i}^{0},
\end{array}\right.
$$

where $\mathcal{A}_{i}$ is the linear operator defined by

$$
\begin{aligned}
& \mathcal{A}_{1} U_{1}=\left(\begin{array}{l}
\varphi_{t} \\
\psi_{t} \\
w_{t} \\
\frac{k_{1}}{\rho_{1}} \varphi_{x x}-\frac{l^{2} k_{3}}{\rho_{1}} \varphi+\frac{k_{1}}{\rho_{1}} \psi_{x}+\frac{l}{\rho_{1}}\left(k_{1}+k_{3}\right) w_{x} \\
-\frac{k_{1}}{\rho_{2}} \varphi_{x}+\frac{1}{\rho_{2}}\left(k_{2}-g_{2}^{0}\right) \psi_{x x}-\frac{k_{1}}{\rho_{2}} \psi-\frac{l k_{1}}{\rho_{2}} w+\frac{1}{\rho_{2}} \int_{0}^{+\infty} g_{2} \partial_{x x} \eta_{2} \mathrm{~d} s \\
-\frac{l}{\rho_{1}}\left(k_{1}+k_{3}\right) \varphi_{x}-\frac{l k_{1}}{\rho_{1}} \psi+\frac{1}{\rho_{1}}\left(k_{3}-g_{3}^{0}\right) w_{x x}-\frac{l^{2} k_{1}}{\rho_{1}} w+\frac{1}{\rho_{1}} \int_{0}^{+\infty} g_{3} \partial_{x x} \eta_{3} \mathrm{~d} s \\
\psi_{t}-\partial_{s} \eta_{2} \\
w_{t}-\partial_{s} \eta_{3}
\end{array}\right), \\
& \mathcal{A}_{2} U_{2}=\left(\begin{array}{l}
\varphi_{t} \\
\psi_{t} \\
w_{t} \\
\frac{1}{\rho_{1}}\left(k_{1}-g_{1}^{0}\right) \varphi_{x x}-\frac{l^{2} k_{3}}{\rho_{1}} \varphi+\frac{k_{1}}{\rho_{1}} \psi_{x}+\frac{l}{\rho_{1}}\left(k_{1}+k_{3}\right) w_{x}+\frac{1}{\rho_{1}} \int_{0}^{+\infty} g_{1} \partial_{x x} \eta_{1} \mathrm{~d} s \\
-\frac{k_{1}}{\rho_{2}} \varphi_{x}+\frac{k_{2}}{\rho_{2}} \psi_{x x}-\frac{k_{1}}{\rho_{2}} \psi-\frac{l k_{1}}{\rho_{2}} w \\
-\frac{l}{\rho_{1}}\left(k_{1}+k_{3}\right) \varphi_{x}-\frac{l k_{1}}{\rho_{1}} \psi+\frac{1}{\rho_{1}}\left(k_{3}-g_{3}^{0}\right) w_{x x}-\frac{l^{2} k_{1}}{\rho_{1}} w+\frac{1}{\rho_{1}} \int_{0}^{+\infty} g_{3} \partial_{x x} \eta_{3} \mathrm{~d} s \\
\varphi_{t}-\partial_{s} \eta_{1} \\
w_{t}-\partial_{s} \eta_{3}
\end{array}\right)
\end{aligned}
$$


and

$$
\mathcal{A}_{3} U_{3}=\left(\begin{array}{l}
\varphi_{t} \\
\psi_{t} \\
w_{t} \\
\frac{1}{\rho_{1}}\left(k_{1}-g_{1}^{0}\right) \varphi_{x x}-\frac{l^{2} k_{3}}{\rho_{1}} \varphi+\frac{k_{1}}{\rho_{1}} \psi_{x}+\frac{l}{\rho_{1}}\left(k_{1}+k_{3}\right) w_{x}+\frac{1}{\rho_{1}} \int_{0}^{+\infty} g_{1} \partial_{x x} \eta_{1} \mathrm{~d} s \\
-\frac{k_{1}}{\rho_{2}} \varphi_{x}+\frac{1}{\rho_{2}}\left(k_{2}-g_{2}^{0}\right) \psi_{x x}-\frac{k_{1}}{\rho_{2}} \psi-\frac{l k_{1}}{\rho_{2}} w+\frac{1}{\rho_{2}} \int_{0}^{+\infty} g_{2} \partial_{x x} \eta_{2} \mathrm{~d} s \\
-\frac{l}{\rho_{1}}\left(k_{1}+k_{3}\right) \varphi_{x}-\frac{l k_{1}}{\rho_{1}} \psi+\frac{k_{3}}{\rho_{1}} w_{x x}-\frac{l^{2} k_{1}}{\rho_{1}} w \\
\varphi_{t}-\partial_{s} \eta_{1} \\
\psi_{t}-\partial_{s} \eta_{2}
\end{array}\right) .
$$

Here, for $i=1,2,3$,

$$
g_{i}^{0}=\int_{0}^{+\infty} g_{i}(s) \mathrm{d} s
$$

Let

$$
\left\{\begin{array}{l}
L_{1}=\left\{v: \mathbb{R}_{+} \rightarrow H_{0}^{1}(] 0, L[), \int_{0}^{L} \int_{0}^{+\infty} g_{1} v_{x}^{2} \mathrm{~d} s \mathrm{~d} x<+\infty\right\}, \\
L_{2}=\left\{v: \mathbb{R}_{+} \rightarrow H_{*}^{1}(] 0, L[), \int_{0}^{L} \int_{0}^{+\infty} g_{2} v_{x}^{2} \mathrm{~d} s \mathrm{~d} x<+\infty\right\} \\
L_{3}=\left\{v: \mathbb{R}_{+} \rightarrow H_{*}^{1}(] 0, L[), \int_{0}^{L} \int_{0}^{+\infty} g_{3} v_{x}^{2} \mathrm{~d} s \mathrm{~d} x<+\infty\right\}
\end{array}\right.
$$

and

$$
\left\{\begin{array}{l}
\mathcal{H}_{1}=H_{0}^{1}(] 0, L[) \times\left(H_{*}^{1}(] 0, L[)\right)^{2} \times L^{2}(] 0, L[) \times\left(L_{*}^{2}(] 0, L[)\right)^{2} \times L_{2} \times L_{3}, \\
\mathcal{H}_{2}=H_{0}^{1}(] 0, L[) \times\left(H_{*}^{1}(] 0, L[)\right)^{2} \times L^{2}(] 0, L[) \times\left(L_{*}^{2}(] 0, L[)\right)^{2} \times L_{1} \times L_{3}, \\
\mathcal{H}_{3}=H_{0}^{1}(] 0, L[) \times\left(H_{*}^{1}(] 0, L[)\right)^{2} \times L^{2}(] 0, L[) \times\left(L_{*}^{2}(] 0, L[)\right)^{2} \times L_{1} \times L_{2},
\end{array}\right.
$$

where

$$
L_{*}^{2}(] 0, L[)=\left\{v \in L^{2}(] 0, L[), \int_{0}^{L} v \mathrm{~d} x=0\right\}
$$

and

$$
H_{*}^{1}(] 0, L[)=\left\{v \in H^{1}(] 0, L[), \int_{0}^{L} v \mathrm{~d} x=0\right\} .
$$


The domain $D\left(\mathcal{A}_{i}\right)$ of $\mathcal{A}_{i}$ is defined by

$$
\begin{gathered}
D\left(\mathcal{A}_{i}\right)=\left\{V=\left(v_{1}, \ldots, v_{8}\right)^{T} \in \mathcal{H}_{i}, \mathcal{A}_{i} V \in \mathcal{H}_{i}, v_{7}(0)=v_{8}(0)=0, \partial_{x} v_{2}(0)=0,\right. \\
\left.\partial_{x} v_{3}(0)=\partial_{x} v_{2}(L)=\partial_{x} v_{3}(L)=0, \partial_{x} v_{j}(\cdot, 0)=\partial_{x} v_{j}(\cdot, L)=0, j=7,8 \text { if } i=1, j=8 \text { if } i=2,3\right\} ;
\end{gathered}
$$

that is, according to the definition of $\mathcal{H}_{i}$ and $\mathcal{A}_{i}$,

$$
\begin{aligned}
D\left(\mathcal{A}_{i}\right) & =\left\{\left(v_{1}, \ldots, v_{8}\right)^{T} \in \mathcal{H}_{i}:\left(v_{1}, \ldots, v_{6}\right)^{T} \in H_{0}^{1}(] 0, L[) \times\left(H_{*}^{1}(] 0, L[)\right)^{2} \times H_{0}^{1}(] 0, L[) \times\left(H_{*}^{1}(] 0, L[)\right)^{2},\right. \\
v_{7}(0) & \left.=v_{8}(0)=0, \partial_{x} v_{2}(0)=\partial_{x} v_{3}(0)=\partial_{x} v_{2}(L)=\partial_{x} v_{3}(L)=0\right\} \cap D_{i},
\end{aligned}
$$

where

$$
\begin{aligned}
& D_{1}=\left\{\left(v_{1}, \ldots, v_{8}\right)^{T} \in \mathcal{H}_{i}: \partial_{s} v_{7} \in L_{2}, \partial_{s} v_{8} \in L_{3}, v_{1} \in H^{2}(] 0, L[),\right. \\
& \partial_{x} v_{7}(\cdot, 0)=\partial_{x} v_{8}(\cdot, 0)=\partial_{x} v_{7}(\cdot, L)=\partial_{x} v_{8}(\cdot, L)=0, \\
& \left.\left(k_{2}-g_{2}^{0}\right) \partial_{x x} v_{2}+\int_{0}^{+\infty} g_{2} \partial_{x x} v_{7} \mathrm{~d} s \in L_{*}^{2}(] 0, L[),\left(k_{3}-g_{3}^{0}\right) \partial_{x x} v_{3}+\int_{0}^{+} g_{3} \partial_{x x} v_{8} \mathrm{~d} s \in L_{*}^{2}(] 0, L[)\right\}, \\
& D_{2}=\left\{\left(v_{1}, \ldots, v_{8}\right)^{T} \in \mathcal{H}_{i}: \partial_{s} v_{7} \in L_{1}, \partial_{s} v_{8} \in L_{3}, v_{2} \in H^{2}(] 0, L[), \partial_{x} v_{8}(\cdot, 0)=\partial_{x} v_{8}(\cdot, L)=0,\right. \\
& \left.\left(k_{1}-g_{1}^{0}\right) \partial_{x x} v_{1}+\int_{0}^{+\infty} g_{1} \partial_{x x} v_{7} \mathrm{~d} s \in L^{2}(] 0, L[),\left(k_{3}-g_{3}^{0}\right) \partial_{x x} v_{3}+\int_{0}^{+\infty} g_{3} \partial_{x x} v_{8} \mathrm{~d} s \in L_{*}^{2}(] 0, L[)\right\}
\end{aligned}
$$

and

$$
\begin{aligned}
& D_{3}=\left\{\left(v_{1}, \ldots, v_{8}\right)^{T} \in \mathcal{H}_{i}: \partial_{s} v_{7} \in L_{1}, \partial_{s} v_{8} \in L_{2}, v_{3} \in H^{2}(] 0, L[), \partial_{x} v_{8}(\cdot, 0)=\partial_{x} v_{8}(\cdot, L)=0,\right. \\
& \left.\left(k_{1}-g_{1}^{0}\right) \partial_{x x} v_{1}+\int_{0}^{+\infty} g_{1} \partial_{x x} v_{7} \mathrm{~d} s \in L^{2}(] 0, L[),\left(k_{2}-g_{2}^{0}\right) \partial_{x x} v_{2}+\int_{0}^{+\infty} g_{2} \partial_{x x} v_{8} \mathrm{~d} s \in L_{*}^{2}(] 0, L[)\right\} .
\end{aligned}
$$

More generally, for $n \in \mathbb{N}$,

$$
D\left(\mathcal{A}_{i}^{n}\right)= \begin{cases}\mathcal{H}_{i} & \text { if } n=0, \\ D\left(\mathcal{A}_{i}\right) & \text { if } n=1, \\ \left\{V \in D\left(\mathcal{A}_{i}^{n-1}\right), \mathcal{A}_{i} V \in D\left(\mathcal{A}_{i}^{n-1}\right)\right\} & \text { if } n=2,3, \ldots\end{cases}
$$

endowed with the graph norm

$$
\|V\|_{D\left(\mathcal{A}_{i}^{n}\right)}=\sum_{k=0}^{n}\left\|\mathcal{A}_{i}^{k} V\right\|_{\mathcal{H}_{i}}
$$

where $\|\cdot\|_{\mathcal{H}_{i}}$ is defined in (43).

Remark 2.1. By integrating on $] 0, L[$ the second and third equations in (1)-(3), and using the boundary conditions, we obtain

$$
\partial_{t t}\left(\int_{0}^{L} \psi \mathrm{d} x\right)+\frac{k_{1}}{\rho_{2}} \int_{0}^{L} \psi \mathrm{d} x+\frac{l k_{1}}{\rho_{2}} \int_{0}^{L} w \mathrm{~d} x=0
$$


and

$$
\partial_{t t}\left(\int_{0}^{L} w \mathrm{~d} x\right)+\frac{l^{2} k_{1}}{\rho_{1}} \int_{0}^{L} w \mathrm{~d} x+\frac{l k_{1}}{\rho_{1}} \int_{0}^{L} \psi \mathrm{d} x=0 .
$$

Therefore, (22) implies that

$$
\int_{0}^{L} w \mathrm{~d} x=-\frac{\rho_{2}}{l k_{1}} \partial_{t t}\left(\int_{0}^{L} \psi \mathrm{d} x\right)-\frac{1}{l} \int_{0}^{L} \psi \mathrm{d} x .
$$

Substituting (24) into (23), we get

$$
\partial_{t t t t}\left(\int_{0}^{L} \psi \mathrm{d} x\right)+\left(\frac{k_{1}}{\rho_{2}}+\frac{l^{2} k_{1}}{\rho_{1}}\right) \partial_{t t}\left(\int_{0}^{L} \psi \mathrm{d} x\right)=0 .
$$

Let $l_{0}=\sqrt{\frac{k_{1}}{\rho_{2}}+\frac{l^{2} k_{1}}{\rho_{1}}}$. Then, solving (25), we find

$$
\int_{0}^{L} \psi \mathrm{d} x=\tilde{c}_{1} \cos \left(l_{0} t\right)+\tilde{c}_{2} \sin \left(l_{0} t\right)+\tilde{c}_{3} t+\tilde{c}_{4},
$$

where $\tilde{c}_{1}, \ldots, \tilde{c}_{4}$ are real constants. By combining (24) and (26), we get

$$
\int_{0}^{L} w \mathrm{~d} x=\tilde{c}_{1}\left(\frac{\rho_{2} l_{0}^{2}}{l k_{1}}-\frac{1}{l}\right) \cos \left(l_{0} t\right)+\tilde{c}_{2}\left(\frac{\rho_{2} l_{0}^{2}}{l k_{1}}-\frac{1}{l}\right) \sin \left(l_{0} t\right)-\frac{\tilde{c}_{3}}{l} t-\frac{\tilde{c}_{4}}{l} .
$$

Let

$$
\left(\tilde{\psi}_{0}(x), \tilde{w}_{0}(x)\right)= \begin{cases}\left(\psi_{0}(x, 0), w_{0}(x, 0)\right) & \text { in case (1), } \\ \left(\psi_{0}(x), w_{0}(x, 0)\right) & \text { in case (2), } \\ \left(\psi_{0}(x, 0), w_{0}(x)\right) & \text { in case (3). }\end{cases}
$$

Using the initial data of $\psi$ and $w$ in (1)-(3), we see that

$$
\left\{\begin{array}{l}
\tilde{c}_{1}=\frac{k_{1}}{\rho_{2} l_{0}^{2}} \int_{0}^{L} \tilde{\psi}_{0} \mathrm{~d} x+\frac{l k_{1}}{\rho_{2} l_{0}^{2}} \int_{0}^{L} \tilde{w}_{0} \mathrm{~d} x, \\
\tilde{c}_{2}=\frac{k_{1}}{\rho_{2} l_{0}^{3}} \int_{0}^{L} \psi_{1} \mathrm{~d} x+\frac{l k_{1}}{\rho_{2} l_{0}^{3}} \int_{0}^{L} w_{1} \mathrm{~d} x \\
\tilde{c}_{3}=\left(1-\frac{k_{1}}{\rho_{2} l_{0}^{2}}\right) \int_{0}^{L} \psi_{1} \mathrm{~d} x-\frac{l k_{1}}{\rho_{2} l_{0}^{2}} \int_{0}^{L} w_{1} \mathrm{~d} x, \\
\tilde{c}_{4}=\left(1-\frac{k_{1}}{\rho_{2} l_{0}^{2}}\right) \int_{0}^{L} \tilde{\psi}_{0} \mathrm{~d} x-\frac{l k_{1}}{\rho_{2} l_{0}^{2}} \int_{0}^{L} \tilde{w}_{0} \mathrm{~d} x .
\end{array}\right.
$$

Let

$$
\tilde{\psi}=\psi-\frac{1}{L}\left(\tilde{c}_{1} \cos \left(l_{0} t\right)+\tilde{c}_{2} \sin \left(l_{0} t\right)+\tilde{c}_{3} t+\tilde{c}_{4}\right)
$$


and

$$
\tilde{w}=w-\frac{1}{L}\left(\tilde{c}_{1}\left(\frac{\rho_{2} l_{0}^{2}}{l k_{1}}-\frac{1}{l}\right) \cos \left(l_{0} t\right)+\tilde{c}_{2}\left(\frac{\rho_{2} l_{0}^{2}}{l k_{1}}-\frac{1}{l}\right) \sin \left(l_{0} t\right)-\frac{\tilde{c}_{3}}{l} t-\frac{\tilde{c}_{4}}{l}\right) .
$$

Then, from (26) and (27) one can check that

$$
\int_{0}^{L} \tilde{\psi} \mathrm{d} x=\int_{0}^{L} \tilde{w} \mathrm{~d} x=0
$$

and, hence,

$$
\int_{0}^{L} \tilde{\eta}_{2} \mathrm{~d} x=\int_{0}^{L} \tilde{\eta}_{3} \mathrm{~d} x=0
$$

where

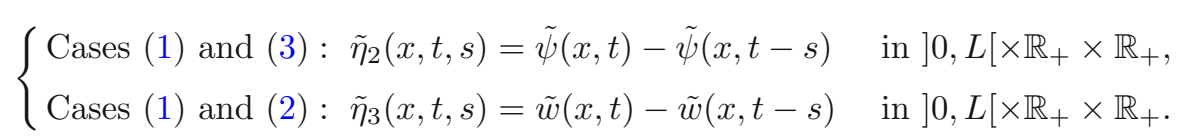

Therefore, the Poincaré's inequality

$$
\exists c_{0}>0: \int_{0}^{L} v^{2} \mathrm{~d} x \leq c_{0} \int_{0}^{L} v_{x}^{2} \mathrm{~d} x, \quad \forall v \in H_{*}^{1}(] 0, L[)
$$

is applicable for $\tilde{\psi}, \tilde{w}, \tilde{\eta}_{2}$ and $\tilde{\eta}_{3}$, provided that $\tilde{\psi}, \tilde{w} \in H^{1}(] 0, L[)$. In addition, $(\varphi, \tilde{\psi}, \tilde{w})$ satisfies the boundary conditions and the first three equations in (1)-(3) with initial data

$$
\begin{gathered}
\psi_{0}-\frac{1}{L}\left(\tilde{c}_{1}+\tilde{c}_{4}\right), \quad \psi_{1}-\frac{1}{L}\left(l_{0} \tilde{c}_{2}+\tilde{c}_{3}\right), \\
w_{0}-\frac{1}{L}\left(\tilde{c}_{1}\left(\frac{\rho_{2} l_{0}^{2}}{l k_{1}}-\frac{1}{l}\right)-\frac{\tilde{c}_{4}}{l}\right) \quad \text { and } w_{1}-\frac{1}{L}\left(\tilde{c}_{2} l_{0}\left(\frac{\rho_{2} l_{0}^{2}}{l k_{1}}-\frac{1}{l}\right)-\frac{\tilde{c}_{3}}{l}\right)
\end{gathered}
$$

instead of $\psi_{0}, \psi_{1}, w_{0}$ and $w_{1}$, respectively. In the sequel, we work with $\tilde{\psi}, \tilde{w}, \tilde{\eta}_{2}$ and $\tilde{\eta}_{3}$ instead of $\psi, w$, $\eta_{2}$ and $\eta_{3}$, but, for simplicity of notation, we use $\psi, w, \eta_{2}$ and $\eta_{3}$ instead of $\tilde{\psi}, \tilde{w}, \tilde{\eta}_{2}$ and $\tilde{\eta}_{3}$, respectively.

Now, to get the well-posedness of (15), we assume the following hypothesis:

(H1) The function $g_{i}: \mathbb{R}_{+} \rightarrow \mathbb{R}_{+}$is differentiable, nonincreasing and integrable on $\mathbb{R}_{+}$such that there exists a positive constant $k_{0}$ such that, for any

$$
(\varphi, \psi, w)^{T} \in H_{0}^{1}(] 0, L[) \times\left(H_{*}^{1}(] 0, L[)\right)^{2},
$$

we have

$$
\begin{aligned}
k_{0} \int_{0}^{L}\left(\varphi_{x}^{2}+\psi_{x}^{2}+w_{x}^{2}\right) \mathrm{d} x \leq \int_{0}^{L}\left(k_{2} \psi_{x}^{2}+k_{1}\left(\varphi_{x}+\psi+l w\right)^{2}+k_{3}\left(w_{x}-l \varphi\right)^{2}\right) \mathrm{d} x \\
\\
-\left\{\begin{array}{l}
\int_{0}^{L}\left(g_{2}^{0} \psi_{x}^{2}+g_{3}^{0} w_{x}^{2}\right) \mathrm{d} x \text { in case }(1), \\
\int_{0}^{L}\left(g_{1}^{0} \varphi_{x}^{2}+g_{3}^{0} w_{x}^{2}\right) \mathrm{d} x \text { in case }(2), \\
\int_{0}^{L}\left(g_{1}^{0} \varphi_{x}^{2}+g_{2}^{0} \psi_{x}^{2}\right) \mathrm{d} x \text { in case }(3) .
\end{array}\right.
\end{aligned}
$$


Moreover, there exists a positive constant $\beta$ such that

$$
-\beta g_{j}(s) \leq g_{j}^{\prime}(s), \quad \forall s \in \mathbb{R}_{+},
$$

where $j \in\{1,2,3\} \backslash\{i\}$ for system $(i), i=1,2,3$.

Remark 2.2. 1. The set of functions satisfying (H1) is very large; indeed, if, for example, the constants $L$ and $l$ satisfy

$$
l L \neq m \pi, \quad \forall m \in \mathbb{N}^{*},
$$

then, by contradiction arguments, we see that there exists a positive constant $\bar{k}_{0}$ such that, for any $(\varphi, \psi, w)^{T} \in H_{0}^{1}(] 0, L[) \times\left(H_{*}^{1}(] 0, L[)\right)^{2}$,

$$
\bar{k}_{0} \int_{0}^{L}\left(\varphi_{x}^{2}+\psi_{x}^{2}+w_{x}^{2}\right) \mathrm{d} x \leq \int_{0}^{L}\left(k_{2} \psi_{x}^{2}+k_{1}\left(\varphi_{x}+\psi+l w\right)^{2}+k_{3}\left(w_{x}-l \varphi\right)^{2}\right) \mathrm{d} x .
$$

To prove (36) in case (35), it is sufficient to prove that, if the right-hand side of (36) vanishes, then

$$
(\varphi, \psi, w)=(0,0,0) .
$$

But (37) can be directly deduced from (30), (35) and the boundary conditions on $\varphi$. Therefore, if (35) holds and

$$
\max _{j \in\{1,2,3\} \backslash\{i\}}\left\{g_{j}^{0}\right\}<\bar{k}_{0} \quad \text { in case }(i), \quad i=1,2,3,
$$

then (33) is satisfied with

$$
k_{0}=\bar{k}_{0}- \begin{cases}\max \left\{g_{2}^{0}, g_{3}^{0}\right\} & \text { in case (1), } \\ \max \left\{g_{1}^{0}, g_{3}^{0}\right\} & \text { in case (2), } \\ \max \left\{g_{1}^{0}, g_{2}^{0}\right\} & \text { in case (3). }\end{cases}
$$

2. Thanks to (32) applied for $\psi$ and $w$, and the Poincaré's inequality

$$
\exists \tilde{c}_{0}>0: \int_{0}^{L} v^{2} \mathrm{~d} x \leq \tilde{c}_{0} \int_{0}^{L} v_{x}^{2} \mathrm{~d} x, \quad \forall v \in H_{0}^{1}(] 0, L[)
$$

applied for $\varphi$, there exists a positive constant $\tilde{k}_{0}$ such that, for any

$$
(\varphi, \psi, w)^{T} \in H_{0}^{1}(] 0, L[) \times\left(H_{*}^{1}(] 0, L[)\right)^{2},
$$

we have

$$
\int_{0}^{L}\left(k_{2} \psi_{x}^{2}+k_{1}\left(\varphi_{x}+\psi+l w\right)^{2}+k_{3}\left(w_{x}-l \varphi\right)^{2}\right) \mathrm{d} x \leq \tilde{k}_{0} \int_{0}^{L}\left(\varphi_{x}^{2}+\psi_{x}^{2}+w_{x}^{2}\right) \mathrm{d} x .
$$

Thus, from (36) and (40), we deduce that the right-hand side of the inequality (36) defines a norm on $H_{0}^{1}(] 0, L[) \times\left(H_{*}^{1}(] 0, L[)\right)^{2}$ for $(\varphi, \psi, w)$ equivalent to the usual norm of $\left(H^{1}(] 0, L[)\right)^{3}$.

3. From (33), we conclude that, in case $(i), i=1,2,3$,

$$
k_{0}+g_{j}^{0}-k_{j} \leq 0, \quad \forall j \in\{1,2,3\} \backslash\{i\} .
$$

Indeed, for the choice $\varphi=w=0,(33)$ in cases (1) and (3) gives

$$
\left(k_{0}+g_{2}^{0}-k_{2}\right) \int_{0}^{L} \psi_{x}^{2} \mathrm{~d} x \leq k_{1} \int_{0}^{L} \psi^{2} \mathrm{~d} x, \quad \forall \psi \in H_{*}^{1}(] 0, L[) .
$$


This inequality implies, for $\psi(x)=\cos (\lambda x)-\frac{1}{\lambda L} \sin (\lambda L)$ and $\left.\lambda \in\right] 0,+\infty\left[\right.$ (notice that $\psi \in H_{*}^{1}(] 0, L[)$ ),

$$
\left(k_{0}+g_{2}^{0}-k_{2}\right)\left(L-\frac{1}{2 \lambda} \sin (2 \lambda L)\right) \leq \frac{k_{1}}{\lambda^{2}}\left(L+\frac{1}{2 \lambda} \sin (2 \lambda L)-\frac{2}{\lambda^{2} L} \sin ^{2}(\lambda L)\right), \quad \forall \lambda>0 .
$$

By letting $\lambda$ go to $+\infty$, we deduce (41), for $j=2$. In the same way, using the choices

$$
(\varphi(x), \psi(x), w(x))=\left(\sin \left(\frac{m \pi}{L} x\right), 0,0\right) \text { and }(\varphi(x), \psi(x), w(x))=\left(0,0, \cos (\lambda x)-\frac{1}{\lambda L} \sin (\lambda L)\right),
$$

for $m \in \mathbb{N}$ and $\lambda \in] 0,+\infty\left[\right.$ (notice that $\varphi \in H_{0}^{1}(] 0, L[)$ and $w \in H_{*}^{1}(] 0, L[)$ ), and letting $m$ and $\lambda$ go to $+\infty$, we conclude (41), for $j=1$ and $j=3$, respectively.

According to Remark 2.2, we notice that, under the hypothesis (H1), the sets $L_{i}$ and $\mathcal{H}_{i}$ are Hilbert spaces equipped, respectively, with the inner products that generate the norms, for $v \in L_{i}$ and $V=$ $\left(v_{1}, \ldots, v_{8}\right)^{T} \in \mathcal{H}_{i}, i=1,2,3$,

$$
\|v\|_{L_{i}}^{2}=\int_{0}^{L} \int_{0}^{+\infty} g_{i} v_{x}^{2} \mathrm{~d} s \mathrm{~d} x
$$

and

$$
\begin{aligned}
\|V\|_{\mathcal{H}_{i}}^{2}= & \int_{0}^{L}\left(k_{2}\left(\partial_{x} v_{2}\right)^{2}+k_{1}\left(\partial_{x} v_{1}+v_{2}+l v_{3}\right)^{2}+k_{3}\left(\partial_{x} v_{3}-l v_{1}\right)^{2}\right) \mathrm{d} x \\
& +\int_{0}^{L}\left(\rho_{1} v_{4}^{2}+\rho_{2} v_{5}^{2}+\rho_{1} v_{6}^{2}\right) \mathrm{d} x+ \begin{cases}\left\|v_{7}\right\|_{L_{2}}^{2}+\left\|v_{8}\right\|_{L_{3}}^{2}-\int_{0}^{L}\left(g_{2}^{0}\left(\partial_{x} v_{2}\right)^{2}+g_{3}^{0}\left(\partial_{x} v_{3}\right)^{2}\right) \mathrm{d} x & \text { if } i=1, \\
\left\|v_{7}\right\|_{L_{1}}^{2}+\left\|v_{8}\right\|_{L_{3}}^{2}-\int_{0}^{L}\left(g_{1}^{0}\left(\partial_{x} v_{1}\right)^{2}+g_{3}^{0}\left(\partial_{x} v_{3}\right)^{2}\right) \mathrm{d} x & \text { if } i=2, \\
\left\|v_{7}\right\|_{L_{1}}^{2}+\left\|v_{8}\right\|_{L_{2}}^{2}-\int_{0}^{L}\left(g_{1}^{0}\left(\partial_{x} v_{1}\right)^{2}+g_{2}^{0}\left(\partial_{x} v_{2}\right)^{2}\right) \mathrm{d} x & \text { if } i=3 .\end{cases}
\end{aligned}
$$

Now, the domain of $D\left(\mathcal{A}_{i}\right)$ is dense in $\mathcal{H}_{i}$, and a simple computation implies that, for any $V=$ $\left(v_{1}, \ldots, v_{8}\right)^{T} \in D\left(\mathcal{A}_{i}\right)$,

$$
\left\langle\mathcal{A}_{i} V, V\right\rangle_{\mathcal{H}_{i}}=\frac{1}{2}\left\{\begin{array}{l}
\int_{0}^{L} \int_{0}^{+\infty}\left(g_{2}^{\prime}\left(\partial_{x} v_{7}\right)^{2}+g_{3}^{\prime}\left(\partial_{x} v_{8}\right)^{2}\right) \mathrm{d} s \mathrm{~d} x \text { if } i=1, \\
\int_{0}^{L+\infty}\left(g_{1}^{\prime}\left(\partial_{x} v_{7}\right)^{2}+g_{3}^{\prime}\left(\partial_{x} v_{8}\right)^{2}\right) \mathrm{d} s \mathrm{~d} x \text { if } i=2, \\
\int_{0}^{L} \int_{0}^{+\infty}\left(g_{1}^{\prime}\left(\partial_{x} v_{7}\right)^{2}+g_{2}^{\prime}\left(\partial_{x} v_{8}\right)^{2}\right) \mathrm{d} s \mathrm{~d} x \text { if } i=3 .
\end{array}\right.
$$

Since $g_{i}$ is nonincreasing, we deduce from (44) that

$$
\left\langle\mathcal{A}_{i} V, V\right\rangle_{\mathcal{H}_{i}} \leq 0
$$


This implies that $A_{i}$ is dissipative. Notice that, according to (34) and the fact that $g_{i}$ is nonincreasing, we see that, for $v \in L_{i}$,

$$
\begin{aligned}
\left|\int_{0}^{L} \int_{0}^{+\infty} g_{i}^{\prime} v_{x}^{2} \mathrm{~d} s \mathrm{~d} x\right| & =-\int_{0}^{L} \int_{0}^{+\infty} g_{i}^{\prime} v_{x}^{2} \mathrm{~d} s \mathrm{~d} x \\
& \leq \beta \int_{0}^{L} \int_{0}^{+\infty} g_{i} v_{x}^{2} \mathrm{~d} s \mathrm{~d} x \\
& \leq \beta\|v\|_{L_{i}}^{2} \\
& <+\infty
\end{aligned}
$$

so the integrals in the right-hand side of (44) are well defined.

Next, we prove that $I d-\mathcal{A}_{i}$ is surjective, where $I d$ is the identity operator. Let $F=\left(f_{1}, \ldots, f_{8}\right)^{T} \in \mathcal{H}_{i}$. We prove the existence of $V=\left(v_{1}, \ldots, v_{8}\right)^{T} \in D\left(\mathcal{A}_{i}\right)$ solution of the equation

$$
\left(I d-\mathcal{A}_{i}\right) V=F
$$

Let us consider the case $\mathcal{A}_{1}$ (the cases $\mathcal{A}_{2}$ and $\mathcal{A}_{3}$ can be treated similarly). The first three equations in (46) reduce to

$$
\left\{\begin{array}{l}
v_{4}=v_{1}-f_{1}, \\
v_{5}=v_{2}-f_{2}, \\
v_{6}=v_{3}-f_{3} .
\end{array}\right.
$$

Using (47), the last two equations in (46) are equivalent to

$$
\left\{\begin{array}{l}
\partial_{s} v_{7}+v_{7}=v_{2}+f_{7}-f_{2}, \\
\partial_{s} v_{8}+v_{8}=v_{3}+f_{8}-f_{3} .
\end{array}\right.
$$

By integrating the two differential equations in (48) and using the fact that $v_{7}(0)=v_{8}(0)=0$ (from (21)), we get

$$
\left\{\begin{array}{l}
v_{7}(s)=\left(1-\mathrm{e}^{-s}\right)\left(v_{2}-f_{2}\right)+\int_{0}^{s} \mathrm{e}^{\tau-s} f_{7}(\tau) \mathrm{d} \tau \\
v_{8}(s)=\left(1-\mathrm{e}^{-s}\right)\left(v_{3}-f_{3}\right)+\int_{0}^{s} \mathrm{e}^{\tau-s} f_{8}(\tau) \mathrm{d} \tau .
\end{array}\right.
$$

We see that, from $(47)$, if $\left(v_{1}, v_{2}, v_{3}\right) \in H_{0}^{1}(] 0, L[) \times\left(H_{*}^{1}(] 0, L[)\right)^{2}$, then $\left(v_{4}, v_{5}, v_{6}\right) \in H_{0}^{1}(] 0, L[) \times$ $\left(H_{*}^{1}(] 0, L[)\right)^{2}$. On the other hand, using Fubini theorem, Hölder's inequality and noting that $f_{7} \in L_{2}$, we get 


$$
\begin{aligned}
\int_{0}^{L} \int_{0}^{+\infty} g_{2}(s)\left(\mathrm{e}^{-s} \int_{0}^{s} \mathrm{e}^{\tau} \partial_{x} f_{7}(\tau) \mathrm{d} \tau\right)^{2} \mathrm{~d} s \mathrm{~d} x & \leq \int_{0}^{L} \int_{0}^{+\infty} \mathrm{e}^{-2 s} g_{2}(s)\left(\int_{0}^{s} \mathrm{e}^{\tau} \mathrm{d} \tau\right) \int_{0}^{s} \mathrm{e}^{\tau}\left(\partial_{x} f_{7}(\tau)\right)^{2} \mathrm{~d} \tau \mathrm{d} s \mathrm{~d} x \\
& \leq \int_{0}^{L} \int_{0}^{+\infty} \mathrm{e}^{-s}\left(1-\mathrm{e}^{-s}\right) g_{2}(s) \int_{0}^{s} \mathrm{e}^{\tau}\left(\partial_{x} f_{7}(\tau)\right)^{2} \mathrm{~d} \tau \mathrm{d} s \mathrm{~d} x \\
& \leq \int_{0}^{L} \int_{0}^{+\infty} \mathrm{e}^{-s} g_{2}(s) \int_{0}^{s} \mathrm{e}^{\tau}\left(\partial_{x} f_{7}(\tau)\right)^{2} \mathrm{~d} \tau \mathrm{d} s \mathrm{~d} x \\
& \leq \int_{0}^{L} \int_{0}^{+\infty} \mathrm{e}^{\tau}\left(\partial_{x} f_{7}(\tau)\right)^{2} \int_{\tau}^{+\infty} \mathrm{e}^{-s} g_{2}(s) \mathrm{d} s \mathrm{~d} \tau \mathrm{d} x \\
& \leq \int_{0}^{L} \int_{0}^{+\infty} \mathrm{e}^{\tau} g_{2}(\tau)\left(\partial_{x} f_{7}(\tau)\right)^{2} \int_{\tau}^{+\infty} \mathrm{e}^{-s} \mathrm{~d} s \mathrm{~d} \tau \mathrm{d} x \\
& \leq \int_{0}^{L} \int_{0}^{+\infty} g_{2}^{+\infty}(\tau)\left(\partial_{x} f_{7}(\tau)\right)^{2} \mathrm{~d} \tau \mathrm{d} x \\
& \leq\left\|f_{7}\right\|_{L_{2}}^{2}<+\infty
\end{aligned}
$$

then

$$
s \mapsto \mathrm{e}^{-s} \int_{0}^{s} \mathrm{e}^{\tau} f_{7}(\tau) \mathrm{d} \tau \in L_{2},
$$

and therefore, (49) implies that $v_{7} \in L_{2}$. Moreover, $\partial_{s} v_{7} \in L_{2}$ since (48). Similarly, we have $v_{8}, \partial_{s} v_{8} \in L_{3}$. So, to prove that (46) (for $i=1$ ) admits a solution $V \in D\left(\mathcal{A}_{1}\right)$, it is enough to prove that

$$
\partial_{x} v_{7}(\cdot, 0)=\partial_{x} v_{8}(\cdot, 0)=\partial_{x} v_{7}(\cdot, L)=\partial_{x} v_{8}(\cdot, L)=0
$$

and $\left(v_{1}, v_{2}, v_{3}\right)$ exists and satisfies the required regularity and boundary conditions in $D\left(\mathcal{A}_{1}\right)$; that is,

$$
\begin{aligned}
& \left(v_{1}, v_{2}, v_{3}\right)^{T} \in\left(H^{2}(] 0, L[) \cap H_{0}^{1}(] 0, L[)\right) \times H_{*}^{1}(] 0, L[) \times H_{*}^{1}(] 0, L[), \\
& \left(k_{2}-g_{2}^{0}\right) \partial_{x x} v_{2}+\int_{0}^{+\infty} g_{2} \partial_{x x} v_{7} \mathrm{~d} s,\left(k_{3}-g_{3}^{0}\right) \partial_{x x} v_{3}+\int_{0}^{+\infty} g_{3} \partial_{x x} v_{8} \mathrm{~d} s \in L_{*}^{2}(] 0, L[)
\end{aligned}
$$

and

$$
\partial_{x} v_{2}(0)=\partial_{x} v_{3}(0)=\partial_{x} v_{2}(L)=\partial_{x} v_{3}(L)=0
$$

Let us assume that (50)-(53) hold. Multiplying the fourth, fifth and sixth equations in (46) by $\rho_{1} \tilde{v}_{1}$, $\rho_{2} \tilde{v}_{2}$ and $\rho_{1} \tilde{v}_{3}$, respectively, integrating their sum over $] 0, L[$, using the boundary conditions (50) and (53), and inserting (47) and (49), we get

$$
a_{1}\left(\left(v_{1}, v_{2}, v_{3}\right)^{T},\left(\tilde{v}_{1}, \tilde{v}_{2}, \tilde{v}_{3}\right)^{T}\right)=\tilde{a}_{1}\left(\left(\tilde{v}_{1}, \tilde{v}_{2}, \tilde{v}_{3}\right)^{T}\right) \text {, }
$$


for any $\left(\tilde{v}_{1}, \tilde{v}_{2}, \tilde{v}_{3}\right)^{T} \in H_{0}^{1}(] 0, L[) \times\left(H_{*}^{1}(] 0, L[)\right)^{2}$, where

$$
\begin{aligned}
& a_{1}\left(\left(v_{1}, v_{2}, v_{3}\right)^{T},\left(\tilde{v}_{1}, \tilde{v}_{2}, \tilde{v}_{3}\right)^{T}\right) \\
& =\int_{0}^{L}\left(k_{1}\left(\partial_{x} v_{1}+v_{2}+l v_{3}\right)\left(\partial_{x} \tilde{v}_{1}+\tilde{v}_{2}+l \tilde{v}_{3}\right)+k_{3}\left(\partial_{x} v_{3}-l v_{1}\right)\left(\partial_{x} \tilde{v}_{3}-l \tilde{v}_{1}\right)\right) \mathrm{d} x \\
& +\int_{0}^{L}\left(\rho_{1} v_{1} \tilde{v}_{1}+\rho_{2} v_{2} \tilde{v}_{2}+\rho_{1} v_{3} \tilde{v}_{3}+\left(k_{2}-\tilde{g}_{2}^{0}\right) \partial_{x} v_{2} \partial_{x} \tilde{v}_{2}-\tilde{g}_{3}^{0} \partial_{x} v_{3} \partial_{x} \tilde{v}_{3}\right) \mathrm{d} x,
\end{aligned}
$$

$\tilde{g}_{i}^{0}=\int_{0}^{+\infty} \mathrm{e}^{-s} g_{i}(s) \mathrm{d} s$ and

$$
\begin{aligned}
\tilde{a}_{1}\left(\left(\tilde{v}_{1}, \tilde{v}_{2}, \tilde{v}_{3}\right)^{T}\right)= & \int_{0}^{L}\left(\rho_{1}\left(f_{1}+f_{4}\right) \tilde{v}_{1}+\rho_{2}\left(f_{2}+f_{5}\right) \tilde{v}_{2}+\rho_{1}\left(f_{3}+f_{6}\right) \tilde{v}_{3}\right) \mathrm{d} x \\
& +\int_{0}^{L}\left(\left(g_{2}^{0}-\tilde{g}_{2}^{0}\right) \partial_{x} f_{2} \partial_{x} \tilde{v}_{2}+\left(g_{3}^{0}-\tilde{g}_{3}^{0}\right) \partial_{x} f_{3} \partial_{x} \tilde{v}_{3}\right) \mathrm{d} x \\
& -\int_{0}^{L}\left(\int_{0}^{+\infty} \mathrm{e}^{-s} g_{2}(s) \int_{0}^{s} \mathrm{e}^{\tau} \partial_{x} f_{7}(\tau) \mathrm{d} \tau \mathrm{d} s\right) \partial_{x} \tilde{v}_{2} \mathrm{~d} x \\
& -\int_{0}^{L}\left(\int_{0}^{+\infty} \mathrm{e}^{-s} g_{3}(s) \int_{0}^{s} \mathrm{e}^{\tau} \partial_{x} f_{8}(\tau) \mathrm{d} \tau \mathrm{d} s\right) \partial_{x} \tilde{v}_{3} \mathrm{~d} x .
\end{aligned}
$$

We remark that, using Fubini's theorem, Hölder's inequality and noting that $f_{7} \in L_{2}$,

$$
\begin{aligned}
\int_{0}^{L}\left(\int_{0}^{+\infty} \mathrm{e}^{-s} g_{2}(s) \int_{0}^{s} \mathrm{e}^{\tau} \partial_{x} f_{7}(\tau) \mathrm{d} \tau \mathrm{d} s\right)^{2} \mathrm{~d} x & \leq \int_{0}^{L}\left(\int_{0}^{+\infty} \mathrm{e}^{-s} g_{2}(s) \int_{0}^{s} \mathrm{e}^{\tau}\left|\partial_{x} f_{7}(\tau)\right| \mathrm{d} \tau \mathrm{d} s\right)^{2} \mathrm{~d} x \\
& \leq \int_{0}^{L}\left(\int_{0}^{+\infty} \mathrm{e}^{\tau}\left|\partial_{x} f_{7}(\tau)\right| \int_{\tau}^{+\infty} g_{2}(s) \mathrm{e}^{-s} \mathrm{~d} s \mathrm{~d} \tau\right)^{2} \mathrm{~d} x \\
& \leq \int_{0}^{L}\left(\int_{0}^{+\infty} g_{2}(\tau) \mathrm{e}^{\tau}\left|\partial_{x} f_{7}(\tau)\right| \int_{\tau}^{+\infty} \mathrm{e}^{-s} \mathrm{~d} s \mathrm{~d} \tau\right)^{2} \mathrm{~d} x \\
& \leq \int_{0}^{L}\left(\int_{0}^{+\infty} g_{2}(\tau)\left|\partial_{x} f_{7}(\tau)\right| \mathrm{d} \tau\right)^{2} \mathrm{~d} x \\
& \leq \int_{0}^{L}\left(\int_{0}^{+\infty} g_{2}(\tau) \mathrm{d} \tau\right)\left(\int_{0}^{+\infty} g_{2}(\tau)\left|\partial_{x} f_{7}(\tau)\right|^{2} \mathrm{~d} \tau\right) \mathrm{d} x \\
& \leq g_{2}^{0}\left\|f_{7}\right\|_{L_{2}}^{2}<+\infty
\end{aligned}
$$


which implies that

$$
x \mapsto \int_{0}^{+\infty} \mathrm{e}^{-s} g_{2}(s) \int_{0}^{s} \mathrm{e}^{\tau} \partial_{x} f_{7}(\tau) \mathrm{d} \tau \mathrm{d} s \in L^{2}(] 0, L[) .
$$

Similarly, we have

$$
x \mapsto \int_{0}^{+\infty} \mathrm{e}^{-s} g_{3}(s) \int_{0}^{s} \mathrm{e}^{\tau} \partial_{x} f_{8}(\tau) \mathrm{d} \tau \mathrm{d} s \in L^{2}(] 0, L[) .
$$

On the other hand, $\tilde{g}_{2}^{0} \leq g_{2}^{0}<k_{2}$ (since (41)) and $\tilde{g}_{3}^{0} \leq g_{3}^{0}$. Then, by virtue of (33) and (40), we have $a_{1}$ is a bilinear, continuous and coercive form on

$$
\left(H_{0}^{1}(] 0, L[) \times\left(H_{*}^{1}(] 0, L[)\right)^{2}\right) \times\left(H_{0}^{1}(] 0, L[) \times\left(H_{*}^{1}(] 0, L[)\right)^{2}\right),
$$

and $\tilde{a}_{1}$ is a linear and continuous form on $H_{0}^{1}(] 0, L[) \times\left(H_{*}^{1}(] 0, L[)\right)^{2}$. Consequently, using Lax-Milgram's theorem, we deduce that (54) has a unique solution

$$
\left(v_{1}, v_{2}, v_{3}\right)^{T} \in H_{0}^{1}(] 0, L[) \times\left(H_{*}^{1}(] 0, L[)\right)^{2} .
$$

Therefore, using classical elliptic regularity arguments, we conclude that the forth, fifth and sixth equations in (46) are satisfied with $\left(v_{1}, v_{2}, v_{3}\right)^{T}$ satisfying (51) and (53), and, using (47) and (49), $v_{7}$ and $v_{8}$ satisfy (50) and (52). Thus, we deduce that (46) (for $i=1$ ) admits a unique solution $V \in D\left(\mathcal{A}_{1}\right)$, and then, $I d-\mathcal{A}_{1}$ is surjective.

Finally, thanks to the Lumer-Phillips theorem (see [19]), we deduce from (45) and (46) that $\mathcal{A}_{i}$ generates a $C_{0}$-semigroup of contractions in $\mathcal{H}_{i}$. This gives the following well-posedness results of (15) (see [13] and [19]).

Theorem 2.3. Assume that (H1) holds. For any $U_{i}^{0} \in D\left(\mathcal{A}_{i}^{n}\right), n \in \mathbb{N}$, (15) has a unique solution

$$
U_{i} \in \cap_{k=0}^{n} C^{n-k}\left(\mathbb{R}_{+} ; D\left(\mathcal{A}_{i}^{k}\right)\right) .
$$

\section{Stability}

In this section, we study the stability of (15), where the obtained two (uniform and weak) decay rates of solution depend on the speeds of wave propagations (4), the smoothness of initial data $U_{i}^{0}$, defined in (13), and the growth of $g_{i}$ at infinity characterized by the following additional hypothesis:

(H2) Assume that $g_{j}(0)>0$, and there exist a positive constant $\alpha$ and an increasing strictly convex function $G: \mathbb{R}_{+} \rightarrow \mathbb{R}_{+}$of class $C^{1}\left(\mathbb{R}_{+}\right) \cap C^{2}(] 0,+\infty[)$ satisfying

$$
G(0)=G^{\prime}(0)=0 \text { and } \lim _{t \rightarrow+\infty} G^{\prime}(t)=+\infty
$$

such that

$$
g_{j}^{\prime}(s) \leq-\alpha g_{j}(s), \quad \forall s \in \mathbb{R}_{+}
$$

or

$$
\int_{0}^{+\infty} \frac{g_{j}(s)}{G^{-1}\left(-g_{j}^{\prime}(s)\right)} \mathrm{d} s+\sup _{s \in \mathbb{R}_{+}} \frac{g_{j}(s)}{G^{-1}\left(-g_{j}^{\prime}(s)\right)}<+\infty,
$$

where $j \in\{1,2,3\} \backslash\{i\}$, for system $(i), i=1,2,3$. 
We start by considering systems (1)-(3) in the case where the speeds of wave propagations (4) satisfy

$$
\begin{cases}s_{1}=s_{2} & \text { in case }(1) \\ s_{2}=s_{1} & \text { in case }(2) \\ s_{3}=s_{1} & \text { in case }(3) .\end{cases}
$$

Theorem 3.1. Assume that (H1), (H2) and (60) $)_{i}$ are satisfied such that

$$
\begin{cases}g_{3}^{0} \text { is small enough } & \text { in case (1), } \\ l<\frac{2 k_{1}}{\sqrt{k_{2} k_{3}}} \text { and } g_{1}^{0} \text { and } g_{3}^{0} \text { are small enough } & \text { in case (2), } \\ g_{1}^{0} \text { is small enough } & \text { in case (3). }\end{cases}
$$

Let $U_{i}^{0} \in \mathcal{H}_{i}$ be such that, for any $j \in\{1,2,3\} \backslash\{i\}$,

$$
\text { (58) holds or } \sup _{t \in \mathbb{R}_{+}} \int_{t}^{+\infty} \frac{g_{j}(s)}{G^{-1}\left(-g_{j}^{\prime}(s)\right)} \int_{0}^{L}\left(\partial_{x} \eta_{j}^{0}(x, s-t)\right)^{2} d x d s<+\infty .
$$

Then, there exist positive constants $c_{i}^{\prime}$ and $c_{i}^{\prime \prime}$ such that the solution of (15) satisfies

$$
\left\|U_{i}(t)\right\|_{\mathcal{H}_{i}}^{2} \leq c_{i}^{\prime \prime} \tilde{G}^{-1}\left(c_{i}^{\prime} t\right), \quad \forall t \in \mathbb{R}_{+},
$$

where $\left.\left.\tilde{G}(s)=\int_{s}^{1} \frac{1}{G_{0}(\tau)} d \tau(s \in] 0,1\right]\right)$ and

$$
G_{0}(s)= \begin{cases}s & \text { if }(58) \text { holds for any } j \in\{1,2,3\} /\{i\} \\ s G^{\prime}(s) & \text { otherwise. }\end{cases}
$$

When $(60)_{i}$ does not hold, we prove the following weaker stability result for (15).

Theorem 3.2. Assume that (H1), (H2) and (61) are satisfied. Let $n \in \mathbb{N}^{*}$ and $U_{i}^{0} \in D\left(\mathcal{A}_{i}^{n}\right)$ be such that, for any $j \in\{1,2,3\} \backslash\{i\}$,

$$
\text { (58) holds or } \sup _{t \in \mathbb{R}_{+}} \max _{k=0, \ldots, n} \int_{t}^{+\infty} \frac{g_{j}(s)}{G^{-1}\left(-g_{j}^{\prime}(s)\right)} \int_{0}^{L}\left(\partial_{s}^{k} \partial_{x} \eta_{j}^{0}(x, s-t)\right)^{2} d x d s<+\infty \text {. }
$$

Then, there exists a positive constant $c_{i, n}$ such that

$$
\left\|U_{i}(t)\right\|_{\mathcal{H}_{i}}^{2} \leq c_{i, n} G_{n}\left(\frac{c_{i, n}}{t}\right), \quad \forall t>0
$$

where $G_{m}(s)=G_{1}\left(s G_{m-1}(s)\right)$, for $m=2, \ldots, n$ and $s \in \mathbb{R}_{+}, G_{1}=G_{0}^{-1}$ and $G_{0}$ is defined in (64).

Remark 3.3. 1. Estimates (63) and (66) imply the strong stability of (15); that is,

$$
\lim _{t \rightarrow+\infty}\left\|U_{i}(t)\right\|_{\mathcal{H}_{i}}^{2}=0 .
$$

2. If (58) holds, for any $j \in\{1,2,3\} \backslash\{i\}$, then (63) and (66) give, respectively,

$$
\left\|U_{i}(t)\right\|_{\mathcal{H}_{i}}^{2} \leq c_{i}^{\prime \prime} \mathrm{e}^{-c_{i}^{\prime} t}, \quad \forall t \in \mathbb{R}_{+}
$$

and

$$
\left\|U_{i}(t)\right\|_{\mathcal{H}_{i}}^{2} \leq \frac{c_{i, n}}{t^{n}}, \quad \forall t>0
$$

The estimates (68) and (69) give the best decay rates which can be obtained from (63) and (66), respectively.

3. Condition (58) implies that $g_{j}$ converges exponentially to zero at infinity. However, condition (59) (introduced in [10]) allows $s \mapsto g_{j}(s)$ to have a decay rate arbitrarily close to $\frac{1}{s}$, which represents the 
critical limit, since $g_{j}$ is integrable on $\mathbb{R}_{+}$. For specific examples of $g_{j}$ and $\eta_{j}$ satisfying (59), (62) and (65), and the corresponding decay rates given by (63) and (66), see [10] and [11].

4. The abstract systems considered in [10] and [11] do not include (1)-(3) because the operator $B$ in [10] is assumed to be positive definite, and the operator $\tilde{B}$ in [11] is assumed to be bounded.

To prove (63) and (66), we will consider suitable multipliers and construct Lyapunov functionals satisfying some differential inequalities, for any $U_{i}^{0} \in D\left(\mathcal{A}_{i}\right)$ and $t \in \mathbb{R}_{+}$; so all the calculations are justified. By integrating these differential inequalities, we get (63) and (66) (for $n=1$ ). By simple density arguments $\left(D\left(\mathcal{A}_{i}\right)\right.$ is dense in $\left.\mathcal{H}_{i}\right)$ and induction on $n,(63)$ remains valid, for any $U_{i}^{0} \in \mathcal{H}_{i}$, and (66) holds, for any $n \in \mathbb{N}^{*}$.

We will use $c$, throughout the rest of this paper, to denote a generic positive constant which depends continuously on the initial data $U_{i}^{0}$ and the fixed parameters in (1)-(3), (32) and (39) and can be different from line to line. When $c$ depends on some new constants $y_{1}, y_{2}, \ldots$, introduced in the proof, the constant $c$ is noted $c_{y_{1}}, c_{y_{1}, y_{2}}, \ldots$

Let us consider the energy functional $E_{i}$ associated with (15) defined by

$$
E_{i}(t)=\frac{1}{2}\left\|U_{i}(t)\right\|_{\mathcal{H}_{i}}^{2} .
$$

From (15) and (44), we see that

$$
E_{i}^{\prime}(t)=\frac{1}{2} \begin{cases}\int_{0}^{L} \int_{0}^{+\infty}\left(g_{2}^{\prime}\left(\partial_{x} \eta_{2}\right)^{2}+g_{3}^{\prime}\left(\partial_{x} \eta_{3}\right)^{2}\right) \mathrm{d} s \mathrm{~d} x & \text { if } i=1, \\ \int_{0}^{L} \int_{0}^{+\infty}\left(g_{1}^{\prime}\left(\partial_{x} \eta_{1}\right)^{2}+g_{3}^{\prime}\left(\partial_{x} \eta_{3}\right)^{2}\right) \mathrm{d} s \mathrm{~d} x & \text { if } i=2, \\ \int_{0}^{L} \int_{0}^{+\infty}\left(g_{1}^{\prime}\left(\partial_{x} \eta_{1}\right)^{2}+g_{2}^{\prime}\left(\partial_{x} \eta_{2}\right)^{2}\right) \mathrm{d} s \mathrm{~d} x & \text { if } i=3 .\end{cases}
$$

Recalling that $g_{i}$ is nonincreasing, (71) implies that $E_{i}$ is nonincreasing, and consequently, (15) is dissipative. If no infinite memory is considered, then $E_{i}^{\prime} \equiv 0$; thus, (15) is a conservative system. This fact shows that the infinite memories generate the unique dissipation in (15). On the other hand, if $E_{i}\left(t_{0}\right)=0$, for some $t_{0} \in \mathbb{R}_{+}$, then $E_{i}(t)=0$, for all $t \geq t_{0}$, and therefore, (63) and (66) hold. Consequently, without loss of generality, we can assume that $E_{i}(t)>0$, for all $t \in \mathbb{R}_{+}$.

\section{Proof of uniform decay (63)}

First, we consider the following functionals:

$$
\begin{aligned}
& \text { Cases (2) and (3): } \quad I_{1}(t)=-\rho_{1} \int_{0}^{L} \varphi_{t} \int_{0}^{+\infty} g_{1}(s) \eta_{1} \mathrm{~d} s \mathrm{~d} x, \\
& \text { Cases (1) and (3): } \quad I_{2}(t)=-\rho_{2} \int_{0}^{L} \psi_{t} \int_{0}^{+\infty} g_{2}(s) \eta_{2} \mathrm{~d} s \mathrm{~d} x
\end{aligned}
$$

and

$$
\text { Cases (1) and (2): } \quad I_{3}(t)=-\rho_{1} \int_{0}^{L} w_{t} \int_{0}^{+\infty} g_{3}(s) \eta_{3} \mathrm{~d} s \mathrm{~d} x \text {. }
$$


Lemma 4.1. For any $\delta_{0}>0$, there exists $c_{\delta_{0}}>0$ such that

$$
\begin{aligned}
I_{1}^{\prime}(t) \leq & -\rho_{1}\left(g_{1}^{0}-\delta_{0}\right) \int_{0}^{L} \varphi_{t}^{2} d x+\delta_{0} \int_{0}^{L}\left(\psi_{x}^{2}+\left(\varphi_{x}+\psi+l w\right)^{2}+\left(w_{x}-l \varphi\right)^{2}\right) d x \\
& +c_{\delta_{0}} \int_{0}^{L} \int_{0}^{+\infty}\left(g_{1}(s)-g_{1}^{\prime}(s)\right)\left(\partial_{x} \eta_{1}\right)^{2} d s d x, \\
I_{2}^{\prime}(t) \leq & -\rho_{2}\left(g_{2}^{0}-\delta_{0}\right) \int_{0}^{L} \psi_{t}^{2} d x+\delta_{0} \int_{0}^{L}\left(\psi_{x}^{2}+\left(\varphi_{x}+\psi+l w\right)^{2}\right) d x \\
& +c_{\delta_{0}} \int_{0}^{L} \int_{0}^{+\infty}\left(g_{2}(s)-g_{2}^{\prime}(s)\right)\left(\partial_{x} \eta_{2}\right)^{2} d s d x
\end{aligned}
$$

and

$$
\begin{aligned}
I_{3}^{\prime}(t) \leq & -\rho_{1}\left(g_{3}^{0}-\delta_{0}\right) \int{ }_{0}^{L} w_{t}^{2} d x+\delta_{0} \int_{0}^{L}\left(\psi_{x}^{2}+\left(\varphi_{x}+\psi+l w\right)^{2}+\left(w_{x}-l \varphi\right)^{2}\right) d x \\
& +c_{\delta_{0}} \int_{0}^{L} \int_{0}^{+\infty}\left(g_{3}(s)-g_{3}^{\prime}(s)\right)\left(\partial_{x} \eta_{3}\right)^{2} d s d x .
\end{aligned}
$$

Proof. First, noticing that

$$
\begin{aligned}
\partial_{t} \int_{0}^{+\infty} g_{1}(s) \eta_{1} \mathrm{~d} s & =\partial_{t} \int_{-\infty}^{t} g_{1}(t-s)(\varphi(t)-\varphi(s)) \mathrm{d} s \\
& =\int_{-\infty}^{t} g_{1}^{\prime}(t-s)(\varphi(t)-\varphi(s)) \mathrm{d} s+\left(\int_{-\infty}^{t} g_{1}(t-s) \mathrm{d} s\right) \varphi_{t}
\end{aligned}
$$

that is,

$$
\partial_{t} \int_{0}^{+\infty} g_{1}(s) \eta_{1} \mathrm{~d} s=\int_{0}^{+\infty} g_{1}^{\prime}(s) \eta_{1} \mathrm{~d} s+g_{1}^{0} \varphi_{t}
$$

Similarly,

$$
\partial_{t} \int_{0}^{+\infty} g_{2}(s) \eta_{2} \mathrm{~d} s=\int_{0}^{+\infty} g_{2}^{\prime}(s) \eta_{2} \mathrm{~d} s+g_{2}^{0} \psi_{t}
$$

and

$$
\partial_{t} \int_{0}^{+\infty} g_{3}(s) \eta_{3} \mathrm{~d} s=\int_{0}^{+\infty} g_{3}^{\prime}(s) \eta_{3} \mathrm{~d} s+g_{3}^{0} w_{t} .
$$

Second, using Young's and Hölder's inequalities, we get the following: For all $\lambda>0$, there exists $c_{\lambda}>0$ such that, for any $v \in L^{2}(] 0, L[)$ and $\eta \in\left\{\eta_{i}, \partial_{x} \eta_{i}\right\}, i=1,2,3$,

$$
\left|\int_{0}^{L} v \int_{0}^{+\infty} g_{i}(s) \eta \mathrm{d} s \mathrm{~d} x\right| \leq \lambda \int_{0}^{L} v^{2} \mathrm{~d} x+c_{\lambda} \int_{0}^{L} \int_{0}^{+\infty} g_{i}(s) \eta^{2} \mathrm{~d} s \mathrm{~d} x .
$$


Similarly,

$$
\left|\int_{0}^{L} v \int_{0}^{+\infty} g_{i}^{\prime}(s) \eta \mathrm{d} s \mathrm{~d} x\right| \leq \lambda \int_{0}^{L} v^{2} \mathrm{~d} x-c_{\lambda} \int_{0}^{L} \int_{0}^{+\infty} g_{i}^{\prime}(s) \eta^{2} \mathrm{~d} s \mathrm{~d} x .
$$

Now, direct computations, using the first equation in (2) or (3), integrating by parts and using the boundary conditions and (78), yield

$$
\begin{aligned}
I_{1}^{\prime}(t)= & -\rho_{1} g_{1}^{0} \int_{0}^{L} \varphi_{t}^{2} \mathrm{~d} x+\int_{0}^{L}\left(\int_{0}^{+\infty} g_{1}(s) \partial_{x} \eta_{1} \mathrm{~d} s\right)^{2} \mathrm{~d} x \\
& +k_{1} \int_{0}^{L}\left(\varphi_{x}+\psi+l w\right) \int_{0}^{+\infty} g_{1}(s) \partial_{x} \eta_{1} \mathrm{~d} s \mathrm{~d} x-l k_{3} \int_{0}^{L}\left(w_{x}-l \varphi\right) \int_{0}^{+\infty} g_{1}(s) \eta_{1} \mathrm{~d} s \mathrm{~d} x \\
& -\rho_{1} \int_{0}^{L} \varphi_{t} \int_{0}^{+\infty} g_{1}^{\prime}(s) \eta_{1} \mathrm{~d} s \mathrm{~d} x-g_{1}^{0} \int_{0}^{L} \varphi_{x} \int_{0}^{+\infty} g_{1}(s) \partial_{x} \eta_{1} \mathrm{~d} s \mathrm{~d} x .
\end{aligned}
$$

Using (81) and (82) for the last four terms of this equality, Poincaré's inequality (39) for $\eta_{1}$ and (36) and Hölder's inequality to estimate

$$
\int_{0}^{L} \varphi_{x}^{2} \mathrm{~d} x \text { and }\left(\int_{0}^{+\infty} g_{1}(s) \partial_{x} \eta_{1} \mathrm{~d} s\right)^{2}
$$

respectively, we get (75).

Similarly, using the second equation in (1) or (3), the third equation in (1) or (2), (79), (80) and (32) (instead of (39)), we find (76) and (77).

Lemma 4.2. Let

$$
\text { Case (1): } \begin{aligned}
J_{1}(t)= & \rho_{2} \int_{0}^{L}\left(\varphi_{x}+\psi+l w\right) \psi_{t} d x+\frac{k_{2} \rho_{1}}{k_{1}} \int_{0}^{L} \psi_{x} \varphi_{t} d x \\
& -\frac{\rho_{1}}{k_{1}} \int_{0}^{L} \varphi_{t} \int_{0}^{+\infty} g_{2}(s) \psi_{x}(t-s)
\end{aligned}
$$

$h b o x d s d x$,

Case (2) : $\quad J_{2}(t)=-\rho_{2} \int_{0}^{L}\left(\varphi_{x}+\psi+l w\right) \psi_{t} d x-\frac{k_{2} \rho_{1}}{k_{1}} \int_{0}^{L} \psi_{x} \varphi_{t} d x$

$h b o x d s d x$

$$
+\frac{\rho_{2}}{k_{1}} \int_{0}^{L} \psi_{t} \int_{0}^{+\infty} g_{1}(s) \varphi_{x}(t-s)
$$


and

$$
\text { Case (3): } \begin{aligned}
J_{3}(t)= & -\rho_{1} \int_{0}^{L}\left(\varphi_{x}+\psi+l w\right) w_{t} d x-\frac{k_{3} \rho_{1}}{k_{1}} \int_{0}^{L}\left(w_{x}-l \varphi\right) \varphi_{t} d x \\
& +\frac{\rho_{1}}{k_{1}} \int_{0}^{L} w_{t} \int_{0}^{+\infty} g_{1}(s) \varphi_{x}(t-s) d x .
\end{aligned}
$$

Then, for any $\delta_{0}, \epsilon_{0}, \epsilon_{1}, \epsilon_{2}>0$, there exists $c_{\delta_{0}}, c_{\epsilon_{0}}>0$ such that

$$
\begin{aligned}
J_{1}^{\prime}(t) \leq & -k_{1} \int_{0}^{L}\left(\varphi_{x}+\psi+l w\right)^{2} d x+\left(\delta_{0}+\frac{l k_{3} \epsilon_{1}}{2 k_{1}}\left(k_{2}-g_{2}^{0}\right)\right) \int_{0}^{L}\left(w_{x}-l \varphi\right)^{2} d x \\
& +\delta_{0} \int_{0}^{L} \varphi_{t}^{2} d x+\frac{l k_{3}}{2 \epsilon_{1} k_{1}}\left(k_{2}-g_{2}^{0}\right) \int_{0}^{L} \psi_{x}^{2} d x+\int_{0}^{L}\left(\frac{3 \rho_{2}}{2} \psi_{t}^{2}+\frac{l^{2} \rho_{2}}{2} w_{t}^{2}\right) d x \\
& +\left(\rho_{2}-\frac{k_{2} \rho_{1}}{k_{1}}\right) \int_{0}^{L} \psi_{t} \varphi_{x t} d x+c_{\delta_{0}} \int_{0}^{L} \int_{0}^{+\infty}\left(g_{2}(s)-g_{2}^{\prime}(s)\right)\left(\partial_{x} \eta_{2}\right)^{2} d s d x \\
J_{2}^{\prime}(t) \leq & \left(k_{1}+\delta_{0}+\frac{g_{1}^{0} \epsilon_{1}}{2}\right) \int_{0}^{L}\left(\varphi_{x}+\psi+l w\right)^{2} d x+\frac{l k_{2} k_{3} \epsilon_{2}}{2 k_{1}} \int_{0}^{L}\left(w_{x}-l \varphi\right)^{2} d x \\
& +\frac{g_{1}^{0}}{2 \epsilon_{1}} \int_{0}^{L} \varphi_{x}^{2} d x+\frac{l k_{2} k_{3}}{2 k_{1} \epsilon_{2}} \int_{0}^{L} \psi_{x}^{2} d x+\left(-\rho_{2}+\delta_{0}+\epsilon_{0}\right) \int_{0}^{L} \psi_{t}^{2} d x+c_{\epsilon_{0}} \int_{0}^{L} w_{t}^{2} d x \\
& +\left(\frac{k_{2} \rho_{1}}{k_{1}}-\rho_{2}\right) \int_{0}^{L} \psi_{t} \varphi_{x t} d x+c_{\delta_{0}} \int_{0}^{L} \int_{0}^{+\infty}\left(g_{1}(s)-g_{1}^{\prime}(s)\right)\left(\partial_{x} \eta_{1}\right)^{2} d s d x
\end{aligned}
$$

and

$$
\begin{aligned}
J_{3}^{\prime}(t) \leq & \left(l k_{1}+\delta_{0}+\frac{l g_{1}^{0} \epsilon_{1}}{2}\right) \int_{0}^{L}\left(\varphi_{x}+\psi+l w\right)^{2} d x-\frac{l k_{3}^{2}}{k_{1}} \int_{0}^{L}\left(w_{x}-l \varphi\right)^{2} d x \\
& +\frac{l g_{1}^{0}}{2 \epsilon_{1}} \int_{0}^{L} \varphi_{x}^{2} d x+c_{\epsilon_{0}} \int_{0}^{L}\left(\varphi_{t}^{2}+\psi_{t}^{2}\right) d x+\left(-l \rho_{1}+\delta_{0}+\epsilon_{0}\right) \int_{0}^{L} w_{t}^{2} d x \\
& +\rho_{1}\left(\frac{k_{3}}{k_{1}}-1\right) \int_{0}^{L} w_{t} \varphi_{x t} d x+c_{\delta_{0}} \int_{0}^{L} \int_{0}^{+\infty}\left(g_{1}(s)-g_{1}^{\prime}(s)\right)\left(\partial_{x} \eta_{1}\right)^{2} d s d x .
\end{aligned}
$$


Proof. First, notice that

$$
\begin{aligned}
\partial_{t} \int_{0}^{+\infty} g_{1}(s) \varphi_{x}(t-s) \mathrm{d} s & =\partial_{t} \int_{-\infty}^{t} g_{1}(t-s) \varphi_{x}(s) \mathrm{d} s \\
& =g_{1}(0) \varphi_{x}(t)+\int_{-\infty}^{t} g_{1}^{\prime}(t-s) \varphi_{x}(s) \mathrm{d} s \\
& =-\int_{0}^{+\infty} g_{1}^{\prime}(s) \varphi_{x}(t) \mathrm{d} s+\int_{0}^{+\infty} g_{1}^{\prime}(s) \varphi_{x}(t-s) \mathrm{d} s
\end{aligned}
$$

that is,

$$
\partial_{t} \int_{0}^{+\infty} g_{1}(s) \varphi_{x}(t-s) \mathrm{d} s=-\int_{0}^{+\infty} g_{1}^{\prime}(s) \partial_{x} \eta_{1} \mathrm{~d} s .
$$

Similarly,

$$
\partial_{t} \int_{0}^{+\infty} g_{2}(s) \psi_{x}(t-s) \mathrm{d} s=-\int_{0}^{+\infty} g_{2}^{\prime}(s) \partial_{x} \eta_{2} \mathrm{~d} s .
$$

Now, by exploiting the first two equations in (1), integrating by parts, recalling (90) and using the boundary conditions, we get

$$
\begin{aligned}
J_{1}^{\prime}(t)= & -k_{1} \int_{0}^{L}\left(\varphi_{x}+\psi+l w\right)^{2} \mathrm{~d} x+\left(\rho_{2}-\frac{k_{2} \rho_{1}}{k_{1}}\right) \int_{0}^{L} \psi_{t} \varphi_{x t} \mathrm{~d} x \\
& +\rho_{2} \int_{0}^{L} \psi_{t}^{2} \mathrm{~d} x+\rho_{2} l \int_{0}^{L} \psi_{t} w_{t} \mathrm{~d} x+\frac{l k_{3}}{k_{1}}\left(k_{2}-g_{2}^{0}\right) \int_{0}^{L}\left(w_{x}-l \varphi\right) \psi_{x} \mathrm{~d} x \\
& +\frac{\rho_{1}}{k_{1}} \int_{0}^{L} \varphi_{t} \int_{0}^{+\infty} g_{2}^{\prime}(s) \partial_{x} \eta_{2} \mathrm{~d} s \mathrm{~d} x+\frac{l k_{3}}{k_{1}} \int_{0}^{L}\left(w_{x}-l \varphi\right) \int_{0}^{+\infty} g_{2}(s) \partial_{x} \eta_{2} \mathrm{~d} s \mathrm{~d} x .
\end{aligned}
$$

By applying (81), (82) and the Young's inequality for the last four terms of the above equality and noting that $k_{2}-g_{2}^{0}>0$ (by virtue of (41)), we deduce (86).

Similarly, using the first two equations in (2), and the first and third equations in (3), and exploiting (89), we find

$$
\begin{aligned}
J_{2}^{\prime}(t)= & k_{1} \int_{0}^{L}\left(\varphi_{x}+\psi+l w\right)^{2} \mathrm{~d} x-g_{1}^{0} \int_{0}^{L}\left(\varphi_{x}+\psi+l w\right) \varphi_{x} \mathrm{~d} x+\left(\frac{k_{2} \rho_{1}}{k_{1}}-\rho_{2}\right) \int_{0}^{L} \psi_{t} \varphi_{x t} \mathrm{~d} x \\
& -\rho_{2} \int_{0}^{L} \psi_{t}^{2} \mathrm{~d} x-l \rho_{2} \int_{0}^{L} \psi_{t} w_{t} \mathrm{~d} x-\frac{l k_{2} k_{3}}{k_{1}} \int_{0}^{L}\left(w_{x}-l \varphi\right) \psi_{x} \mathrm{~d} x \\
& -\frac{\rho_{2}}{k_{1}} \int_{0}^{L} \psi_{t} \int_{0}^{+\infty} g_{1}^{\prime}(s) \partial_{x} \eta_{1} \mathrm{~d} s \mathrm{~d} x+\int_{0}^{L}\left(\varphi_{x}+\psi+l w\right) \int_{0}^{+\infty} g_{1}(s) \partial_{x} \eta_{1} \mathrm{~d} s \mathrm{~d} x
\end{aligned}
$$


and

$$
\begin{aligned}
J_{3}^{\prime}(t)= & l k_{1} \int_{0}^{L}\left(\varphi_{x}+\psi+l w\right)^{2} \mathrm{~d} x-l g_{1}^{0} \int_{0}^{L}\left(\varphi_{x}+\psi+l w\right) \varphi_{x} \mathrm{~d} x+\rho_{1}\left(\frac{k_{3}}{k_{1}}-1\right) \int_{0}^{L} w_{t} \varphi_{x t} \mathrm{~d} x \\
& -\frac{l k_{3}^{2}}{k_{1}} \int_{0}^{L}\left(w_{x}-l \varphi\right)^{2} \mathrm{~d} x-\int_{0}^{L}\left(l \rho_{1} w_{t}^{2}+\rho_{1} \psi_{t} w_{t}-\frac{l \rho_{1} k_{3}}{k_{1}} \varphi_{t}^{2}\right) \mathrm{d} x \\
& -\frac{\rho_{1}}{k_{1}} \int_{0}^{L} w_{t} \int_{0}^{+\infty} g_{1}^{\prime}(s) \partial_{x} \eta_{1} \mathrm{~d} s \mathrm{~d} x+l \int_{0}^{L}\left(\varphi_{x}+\psi+l w\right) \int_{0}^{+\infty} g_{1}(s) \partial_{x} \eta_{1} \mathrm{~d} s \mathrm{~d} x .
\end{aligned}
$$

Then, by proceeding as for (86), we conclude (87) and (88).

Lemma 4.3. Let

$$
D(t)= \begin{cases}0 & \text { in cases (1) and (3), } \\ \rho_{2} \int_{0}^{L} \psi_{x} \int_{0}^{x} \psi_{t}(y, t) d y d x & \text { in case (2). }\end{cases}
$$

Then, for any $\delta_{1}>0$, we have

$$
D^{\prime}(t) \leq-\rho_{2} \int_{0}^{L} \psi_{t}^{2} d x+\left(\frac{k_{1} \delta_{1}}{2}+k_{2}\right) \int_{0}^{L} \psi_{x}^{2} d x+\frac{k_{1} \tilde{c}_{0}}{2 \delta_{1}} \int_{0}^{L}\left(\varphi_{x}+\psi+l w\right)^{2} d x
$$

in case (2), and $D^{\prime}(t)=0$ in cases (1) and (3).

Proof. By exploiting the second equation in (2), integrating by parts and using the boundary conditions, we get

$$
D^{\prime}(t)=\int_{0}^{L}\left(-\rho_{2} \psi_{t}^{2}+k_{2} \psi_{x}^{2}\right) \mathrm{d} x-k_{1} \int_{0}^{L} \psi_{x} \int_{0}^{x}\left(\varphi_{x}(y, t)+\psi(y, t)+l w(y, t)\right) \mathrm{d} y \mathrm{~d} x
$$

in case (2), and $D^{\prime}(t)=0$ in cases (1) and (3). Now, noting that the function

$$
x \mapsto \int_{0}^{x}\left(\varphi_{x}(y, t)+\psi(y, t)+l w(y, t)\right) \mathrm{d} y
$$

vanishes at 0 and $L$ (because of (30)), then, applying (39), we have

$$
\int_{0}^{L}\left(\int_{0}^{x}\left(\varphi_{x}(y, t)+\psi(y, t)+l w(y, t)\right) \mathrm{d} y\right)^{2} \mathrm{~d} x \leq \tilde{c}_{0} \int_{0}^{L}\left(\varphi_{x}+\psi+l w\right)^{2} \mathrm{~d} x .
$$

By applying Young's inequality for the last term in (93) and recalling (94), we conclude (92). 
Lemma 4.4. Let

$$
\text { Case (1): } \begin{aligned}
P_{1}(t)= & -\rho_{1} k_{3} \int_{0}^{L}\left(w_{x}-l \varphi\right) \int_{0}^{x} w_{t}(y, t) d y d x \\
& -\rho_{1} k_{1} \int_{0}^{L} \varphi_{t} \int_{0}^{x}\left(\varphi_{x}+\psi+l w\right)(y, t) d y d x,
\end{aligned}
$$

Case (2): $\quad P_{2}(t)=P_{1}(t)$

and

$$
\text { Case (3): } \quad P_{3}(t)=-P_{1}(t) .
$$

Then, for any $\epsilon_{0}, \delta_{0}, \delta_{1}, \delta_{2}, \delta_{3}>0$, there exists $c_{\epsilon_{0}}, c_{\delta_{0}}>0$ such that

$$
\begin{aligned}
P_{1}^{\prime}(t) \leq & k_{1}^{2} \int_{0}^{L}\left(\varphi_{x}+\psi+l w\right)^{2} d x+\left(\frac{k_{3} g_{3}^{0} \delta_{1}}{2}+\delta_{0}-k_{3}^{2}\right) \int_{0}^{L}\left(w_{x}-l \varphi\right)^{2} d x \\
& +\left(-\rho_{1} k_{1}+\epsilon_{0}\right) \int_{0}^{L} \varphi_{t}^{2} d x+c_{\epsilon_{0}} \int_{0}^{L}\left(\psi_{t}^{2}+w_{t}^{2}\right) d x+\frac{k_{3} g_{3}^{0}}{2 \delta_{1}} \int_{0}^{L} w_{x}^{2} d x \\
& +c_{\delta_{0}} \int_{0}^{L} \int_{0}^{+\infty} g_{3}(s)\left(\partial_{x} \eta_{3}\right)^{2} d s d x \\
P_{2}^{\prime}(t) \leq & \left(k_{1}^{2}+\frac{k_{1} g_{1}^{0} \delta_{2}}{2}+\delta_{0}\right) \int_{0}^{L}\left(\varphi_{x}+\psi+l w\right)^{2} d x+\left(\frac{k_{3} g_{3}^{0} \delta_{3}}{2}+\delta_{0}-k_{3}^{2}\right) \int_{0}^{L}\left(w_{x}-l \varphi\right)^{2} d x \\
& +\epsilon_{0} \int_{0}^{L} \psi_{t}^{2} d x+c_{\epsilon_{0}} \int_{0}^{L}\left(\varphi_{t}^{2}+w_{t}^{2}\right) d x+\frac{k_{1} g_{1}^{0}}{2 \delta_{2}} \int_{0}^{L} \varphi_{x}^{2} d x+\frac{k_{3} g_{3}^{0}}{2 \delta_{3}} \int_{0}^{L} w_{x}^{2} d x \\
& +c_{\delta_{0}} \int_{0}^{L} \int_{0}^{+\infty}\left(g_{1}(s)\left(\partial_{x} \eta_{1}\right)^{2}+g_{3}(s)\left(\partial_{x} \eta_{3}\right)^{2}\right) d s d x
\end{aligned}
$$

and

$$
\begin{aligned}
P_{3}^{\prime}(t) \leq & +\left(\frac{k_{1} g_{1}^{0} \delta_{1}}{2}+\delta_{0}-k_{1}^{2}\right) \int_{0}^{L}\left(\varphi_{x}+\psi+l w\right)^{2} d x+k_{3}^{2} \int_{0}^{L}\left(w_{x}-l \varphi\right)^{2} d x \\
& +\left(-\rho_{1} k_{3}+\epsilon_{0}\right) \int_{0}^{L} w_{t}^{2} d x+c_{\epsilon_{0}} \int_{0}^{L}\left(\varphi_{t}^{2}+\psi_{t}^{2}\right) d x+\frac{k_{1} g_{1}^{0}}{2 \delta_{1}} \int_{0}^{L} \varphi_{x}^{2} d x \\
& +c_{\delta_{0}} \int_{0}^{L} \int_{0}^{+\infty} g_{1}(s)\left(\partial_{x} \eta_{1}\right)^{2} d s d x
\end{aligned}
$$


Proof. By exploiting the first and third equations in (1)-(3), integrating by parts and using (30), (31) and the boundary conditions, we find

$$
\begin{aligned}
& P_{i}^{\prime}(t)=\rho_{1} k_{3} \int_{0}^{L} w_{t}^{2} \mathrm{~d} x-\rho_{1} k_{1} \int_{0}^{L} \varphi_{t}^{2} \mathrm{~d} x-k_{3}^{2} \int_{0}^{L}\left(w_{x}-l \varphi\right)^{2} \mathrm{~d} x \\
& +k_{1}^{2} \int_{0}^{L}\left(\varphi_{x}+\psi+l w\right)^{2} \mathrm{~d} x+l \rho_{1} k_{3} \int_{0}^{L} \varphi_{t} \int_{0}^{x} w_{t}(y, t) \mathrm{d} y \mathrm{~d} x \\
& -\rho_{1} k_{1} \int_{0}^{L} \varphi_{t} \int_{0}^{x}\left(\psi_{t}(y, t)+l w_{t}(y, t)\right) \mathrm{d} y \mathrm{~d} x \\
& +k_{3} g_{3}^{0} \int_{0}^{L}\left(w_{x}-l \varphi\right) w_{x} \mathrm{~d} x-k_{3} \int_{0}^{L}\left(w_{x}-l \varphi\right) \int_{0}^{+\infty} g_{3}(s) \partial_{x} \eta_{3} \mathrm{~d} s \mathrm{~d} x \\
& + \begin{cases}0 & \text { if } i=1, \\
-k_{1} g_{1}^{0} \int_{0}^{L}\left(\varphi_{x}+\psi+l w\right) \varphi_{x} \mathrm{~d} x+k_{1} \int_{0}^{L}\left(\varphi_{x}+\psi+l w\right) \int_{0}^{+\infty} g_{1}(s) \partial_{x} \eta_{1} \mathrm{~d} s \mathrm{~d} x & \text { if } i=2\end{cases}
\end{aligned}
$$

and

$$
\begin{aligned}
P_{3}^{\prime}(t)= & -\rho_{1} k_{3} \int_{0}^{L} w_{t}^{2} \mathrm{~d} x+\rho_{1} k_{1} \int_{0}^{L} \varphi_{t}^{2} \mathrm{~d} x+k_{3}^{2} \int_{0}^{L}\left(w_{x}-l \varphi\right)^{2} \mathrm{~d} x \\
& -k_{1}^{2} \int_{0}^{L}\left(\varphi_{x}+\psi+l w\right)^{2} \mathrm{~d} x-l \rho_{1} k_{3} \int_{0}^{L} \varphi_{t} \int_{0}^{x} w_{t}(y, t) \mathrm{d} y \mathrm{~d} x \\
& +\rho_{1} k_{1} \int_{0}^{L} \varphi_{t} \int_{0}^{x}\left(\psi_{t}(y, t)+l w_{t}(y, t)\right) \mathrm{d} y \mathrm{~d} x \\
& +k_{1} g_{1}^{0} \int_{0}^{L}\left(\varphi_{x}+\psi+l w\right) \varphi_{x} \mathrm{~d} x-k_{1} \int_{0}^{L}\left(\varphi_{x}+\psi+l w\right) \int_{0}^{+\infty} g_{1}(s) \partial_{x} \eta_{1} \mathrm{~d} s \mathrm{~d} x .
\end{aligned}
$$

Now, noting that the functions

$$
x \mapsto \int_{0}^{x} \psi_{t}(y, t) \mathrm{d} y \quad \text { and } \quad x \mapsto \int_{0}^{x} w_{t}(y, t) \mathrm{d} y
$$

vanish at 0 and $L$ (because of (30)), then, applying (39), we have

$$
\int_{0}^{L}\left(\int_{0}^{x} \psi_{t}(y, t) \mathrm{d} y\right)^{2} \mathrm{~d} x \leq \tilde{c}_{0} \int_{0}^{L} \psi_{t}^{2} \mathrm{~d} x
$$

and

$$
\int_{0}^{L}\left(\int_{0}^{x} w_{t}(y, t) \mathrm{d} y\right)^{2} \mathrm{~d} x \leq \tilde{c}_{0} \int_{0}^{L} w_{t}^{2} \mathrm{~d} x .
$$


By applying Young's inequality and (81) in (101) and (102), and recalling (103) and (104), we deduce (98)-(100).

Lemma 4.5. Let

$$
\text { Cases (1)-(3): } \quad R(t)=\int_{0}^{L}\left(\rho_{1} \varphi \varphi_{t}+\rho_{2} \psi \psi_{t}+\rho_{1} w w_{t}\right) d x .
$$

Then, for any $\delta_{0}>0$, there exists $c_{\delta_{0}}>0$ such that

$$
\begin{aligned}
& R^{\prime}(t) \leq \int_{0}^{L}\left(-k_{2} \psi_{x}^{2}-k_{1}\left(\varphi_{x}+\psi+l w\right)^{2}-k_{3}\left(w_{x}-l \varphi\right)^{2}+\rho_{1} \varphi_{t}^{2}+\rho_{2} \psi_{t}^{2}+\rho_{1} w_{t}^{2}\right) d x \\
& +\left\{\begin{array}{l}
\int_{0}^{L}\left(\left(\delta_{0}+g_{2}^{0}\right) \psi_{x}^{2}+\left(\delta_{0}+g_{3}^{0}\right) w_{x}^{2}\right) d x+c_{\delta_{0}} \int_{0}^{L} \int_{0}^{+\infty}\left(g_{2}(s)\left(\partial_{x} \eta_{2}\right)^{2}+g_{3}(s)\left(\partial_{x} \eta_{3}\right)^{2}\right) d s d x \text { in case (1), } \\
\int_{0}^{L}\left(\left(\delta_{0}+g_{1}^{0}\right) \varphi_{x}^{2}+\left(\delta_{0}+g_{3}^{0}\right) w_{x}^{2}\right) d x+c_{\delta_{0}} \int_{0}^{L} \int_{0}^{+\infty}\left(g_{1}(s)\left(\partial_{x} \eta_{1}\right)^{2}+g_{3}(s)\left(\partial_{x} \eta_{3}\right)^{2}\right) d s d x \text { in case (2), } \\
\int_{0}^{L}\left(\left(\delta_{0}+g_{1}^{0}\right) \varphi_{x}^{2}+\left(\delta_{0}+g_{2}^{0}\right) \psi_{x}^{2}\right) d x+c_{\delta_{0}} \int_{0}^{L} \int_{0}^{+\infty}\left(g_{1}(s)\left(\partial_{x} \eta_{1}\right)^{2}+g_{2}(s)\left(\partial_{x} \eta_{2}\right)^{2}\right) d s d x \text { in case (3). }
\end{array}\right.
\end{aligned}
$$

Proof. By using the first three equations in (1)-(3) and the boundary conditions, we obtain

$$
\begin{aligned}
& R^{\prime}(t)=\int_{0}^{L}\left(-k_{2} \psi_{x}^{2}-k_{1}\left(\varphi_{x}+\psi+l w\right)^{2}-k_{3}\left(w_{x}-l \varphi\right)^{2}+\rho_{1} \varphi_{t}^{2}+\rho_{2} \psi_{t}^{2}+\rho_{1} w_{t}^{2}\right) \mathrm{d} x \\
& +\left\{\begin{array}{l}
\int_{0}^{L}\left(g_{2}^{0} \psi_{x}^{2}+g_{3}^{0} w_{x}^{2}\right) \mathrm{d} x-\int_{0}^{L} \psi_{x} \int_{0}^{+\infty} g_{2}(s) \partial_{x} \eta_{2} \mathrm{~d} s \mathrm{~d} x-\int_{0}^{L} w_{x} \int_{0}^{+\infty} g_{3}(s) \partial_{x} \eta_{3} \mathrm{~d} s \mathrm{~d} x \text { in case }(1), \\
\int_{0}^{L}\left(g_{1}^{0} \varphi_{x}^{2}+g_{3}^{0} w_{x}^{2}\right) \mathrm{d} x-\int_{0}^{L} \varphi_{x} \int_{0}^{+\infty} g_{1}(s) \partial_{x} \eta_{1} \mathrm{~d} s \mathrm{~d} x-\int_{0}^{L} w_{x} \int_{0}^{+\infty} g_{3}(s) \partial_{x} \eta_{3} \mathrm{~d} s \mathrm{~d} x \text { in case }(2), \\
\int_{0}^{L}\left(g_{1}^{0} \varphi_{x}^{2}+g_{2}^{0} \psi_{x}^{2}\right) \mathrm{d} x-\int_{0}^{L} \varphi_{x} \int_{0}^{+\infty} g_{1}(s) \partial_{x} \eta_{1} \mathrm{~d} s \mathrm{~d} x-\int_{0}^{+\infty} \psi_{x} g_{0}^{+} g_{2}(s) \partial_{x} \eta_{2} \mathrm{~d} s \mathrm{~d} x \quad \text { in case (3). }
\end{array}\right.
\end{aligned}
$$

By applying (81) for the last two terms in (107), we conclude (106).

Let $N, N_{1}, N_{2}, N_{3}, N_{4} \geq 0$ and, for $i=1,2,3$,

$$
F_{i}:=N E_{i}+J_{i}+N_{2} D+N_{3} P_{i}+N_{4} R+N_{1} \sum_{j \in\{1,2,3\} \backslash\{i\}} I_{j} .
$$


Then, by combining (75)-(77), (86)-(88), (92), (98)-(100) and (106), we obtain

$$
\begin{aligned}
& F_{i}^{\prime}(t) \leq \int_{0}^{L}\left(l_{1} \varphi_{t}^{2}+l_{2} \psi_{t}^{2}+l_{3} w_{t}^{2}+l_{4} \psi_{x}^{2}+\left(l_{5}+g_{3}^{0} \tilde{l}_{5}\right)\left(w_{x}-l \varphi\right)^{2}+\left(l_{6}+g_{1}^{0} \tilde{l}_{6}\right)\left(\varphi_{x}+\psi+l w\right)^{2}\right) \mathrm{d} x \\
& +\int_{0}^{L}\left(g_{1}^{0} l_{7} \varphi_{x}^{2}+g_{3}^{0} l_{8} w_{x}^{2}\right) \mathrm{d} x+N E_{i}^{\prime}(t)+c_{N_{1}, N_{3}, N_{4}, \delta_{0}} \sum_{j \in\{1,2,3\} \backslash\{i\}} \int_{0}^{L} \int_{0}^{+\infty}\left(g_{j}(s)-g_{j}^{\prime}(s)\right)\left(\partial_{x} \eta_{j}\right)^{2} \mathrm{~d} s \mathrm{~d} x \\
& +\delta_{0} c_{N_{1}, N_{3}, N_{4}} \int_{0}^{L}\left(\varphi_{x}^{2}+\psi_{x}^{2}+w_{x}^{2}+\left(\varphi_{x}+\psi+l w\right)^{2}+\left(w_{x}-l \varphi\right)^{2}+\varphi_{t}^{2}+\psi_{t}^{2}+w_{t}^{2}\right) \mathrm{d} x \\
& +\left\{\begin{array}{l}
c_{N_{3}, \epsilon_{0}} \int_{0}^{L}\left(\psi_{t}^{2}+w_{t}^{2}\right) \mathrm{d} x+\epsilon_{0} c_{N_{3}} \int_{0}^{L} \varphi_{t}^{2} \mathrm{~d} x+\left(\rho_{2}-\frac{k_{2} \rho_{1}}{k_{1}}\right) \int_{0}^{L} \psi_{t} \varphi_{x t} \mathrm{~d} x \text { if } i=1, \\
c_{N_{3}, \epsilon_{0}}^{L}\left(\varphi_{t}^{L}+w_{t}^{2}\right) \mathrm{d} x+\epsilon_{0} c_{N_{3}} \int_{0}^{L} \psi_{t}^{2} \mathrm{~d} x+\left(\frac{k_{2} \rho_{1}}{k_{1}}-\rho_{2}\right) \int_{0}^{L} \psi_{t} \varphi_{x t} \mathrm{~d} x \text { if } i=2, \\
c_{N_{3}, \epsilon_{0}}^{L}\left(\varphi_{t}^{L}+\psi_{t}^{2}\right) \mathrm{d} x+\epsilon_{0} c_{N_{3}} \int_{0}^{L} w_{t}^{2} \mathrm{~d} x+\rho_{1}\left(\frac{k_{3}}{k_{1}}-1\right) \int_{0}^{L} w_{t} \varphi_{x t} \mathrm{~d} x \quad \text { if } i=3,
\end{array}\right.
\end{aligned}
$$

where

$$
\begin{aligned}
& l_{1}=\left\{\begin{array}{ll}
-\rho_{1} k_{1} N_{3}+\rho_{1} N_{4} & \text { if } i=1, \\
-\rho_{1} g_{1}^{0} N_{1}+\rho_{1} N_{4} & \text { if } i=2, \\
-\rho_{1} g_{1}^{0} N_{1}+\rho_{1} N_{4} & \text { if } i=3,
\end{array} \quad l_{2}= \begin{cases}-\rho_{2} g_{2}^{0} N_{1}+\rho_{2} N_{4} & \text { if } i=1, \\
-\rho_{2} N_{2}+\rho_{2} N_{4}-\rho_{2} & \text { if } i=2, \\
-\rho_{2} g_{2}^{0} N_{1}+\rho_{2} N_{4} & \text { if } i=3,\end{cases} \right. \\
& l_{3}=\left\{\begin{array}{ll}
-\rho_{1} g_{3}^{0} N_{1}+\rho_{1} N_{4} & \text { if } i=1, \\
-\rho_{1} g_{3}^{0} N_{1}+\rho_{1} N_{4} & \text { if } i=2, \\
-\rho_{1} k_{3} N_{3}+\rho_{1} N_{4}-l \rho_{1} & \text { if } i=3,
\end{array} l_{4}= \begin{cases}-\left(k_{2}-g_{2}^{0}\right) N_{4}+\frac{l k_{3}\left(k_{2}-g_{2}^{0}\right)}{2 k_{1} \epsilon_{1}} & \text { if } i=1, \\
\left(\frac{k_{1} \delta_{1}}{2}+k_{2}\right) N_{2}-k_{2} N_{4}+\frac{l k_{2} k_{3}}{2 k_{1} \epsilon_{2}} & \text { if } i=2, \\
-\left(k_{2}-g_{2}^{0}\right) N_{4} & \text { if } i=3,\end{cases} \right. \\
& l_{5}=\left\{\begin{array}{ll}
-k_{3}^{2} N_{3}-k_{3} N_{4}+\frac{l k_{3}\left(k_{2}-g_{2}^{0}\right) \epsilon_{1}}{2 k_{1}} & \text { if } i=1, \\
-k_{3}^{2} N_{3}-k_{3} N_{4}+\frac{l k_{2} k_{3} \epsilon_{2}}{2 k_{1}} & \text { if } i=2, \\
k_{3}^{2} N_{3}-k_{3} N_{4}-\frac{l k_{3}^{2}}{k_{1}} & \text { if } i=3,
\end{array} \quad \tilde{l}_{5}= \begin{cases}\frac{k_{3} \delta_{1}}{2} N_{3} & \text { if } i=1, \\
\frac{k_{3} \delta_{3}}{2} N_{3} & \text { if } i=2, \\
0 & \text { if } i=3,\end{cases} \right. \\
& l_{6}=\left\{\begin{array}{ll}
k_{1}^{2} N_{3}-k_{1} N_{4}-k_{1} & \text { if } i=1, \\
\frac{k_{1} \tilde{c}_{0}}{2 \delta_{1}} N_{2}+k_{1}^{2} N_{3}-k_{1} N_{4}+k_{1} & \text { if } i=2, \\
-k_{1}^{2} N_{3}-k_{1} N_{4}+l k_{1} & \text { if } i=3,
\end{array} \quad \tilde{l}_{6}= \begin{cases}0 & \text { if } i=1, \\
\frac{k_{1} \delta_{2}}{2} N_{3}+\frac{\epsilon_{1}}{2} & \text { if } i=2, \\
\frac{k_{1} \delta_{1}}{2} N_{3}+\frac{l \epsilon_{1}}{2} & \text { if } i=3,\end{cases} \right. \\
& l_{7}=\left\{\begin{array}{ll}
0 & \text { if } i=1, \\
\frac{k_{1}}{2 \delta_{2}} N_{3}+N_{4}+\frac{1}{2 \epsilon_{1}} & \text { if } i=2, \\
\frac{k_{1}}{2 \delta_{1}} N_{3}+N_{4}+\frac{l}{2 \epsilon_{1}} & \text { if } i=3
\end{array} \quad \text { and } \quad l_{8}= \begin{cases}\frac{k_{3}}{2 \delta_{1}} N_{3}+N_{4} & \text { if } i=1, \\
\frac{k_{3}}{2 \delta_{3}} N_{3}+N_{4} & \text { if } i=2, \\
0 & \text { if } i=3 .\end{cases} \right.
\end{aligned}
$$


Using (33), (43), (70) and (71), we get from (109) that

$$
\begin{aligned}
& F_{i}^{\prime}(t) \leq \int_{0}^{L}\left(l_{1} \varphi_{t}^{2}+l_{2} \psi_{t}^{2}+l_{3} w_{t}^{2}+l_{4} \psi_{x}^{2}+\left(l_{5}+g_{3}^{0} \tilde{l}_{5}\right)\left(w_{x}-l \varphi\right)^{2}+\left(l_{6}+g_{1}^{0} \tilde{l}_{6}\right)\left(\varphi_{x}+\psi+l w\right)^{2}\right) \mathrm{d} x \\
& +\int_{0}^{L}\left(g_{1}^{0} l_{7} \varphi_{x}^{2}+g_{3}^{0} l_{8} w_{x}^{2}\right) \mathrm{d} x+\delta_{0} c_{N_{1}, N_{3}, N_{4}} E_{i}(t) \\
& +\left(N-c_{N_{1}, N_{3}, N_{4}, \delta_{0}}\right) E_{i}^{\prime}(t)+c_{N_{1}, N_{3}, N_{4}, \delta_{0}} \sum_{j \in\{1,2,3\} \backslash\{i\}} \int_{0}^{L} \int_{0}^{+\infty} g_{j}(s)\left(\partial_{x} \eta_{j}\right)^{2} \mathrm{~d} s \mathrm{~d} x \\
& +\left\{\begin{array}{l}
c_{N_{3}, \epsilon_{0}} \int_{0}^{L}\left(\psi_{t}^{2}+w_{t}^{2}\right) \mathrm{d} x+\epsilon_{0} c_{N_{3}} \int_{0}^{L} \varphi_{t}^{2} \mathrm{~d} x+\left(\rho_{2}-\frac{k_{2} \rho_{1}}{k_{1}}\right) \int_{0}^{L} \psi_{t} \varphi_{x t} \mathrm{~d} x \quad \text { if } i=1, \\
c_{N_{3}, \epsilon_{0}} \int_{0}^{L}\left(\varphi_{t}^{2}+w_{t}^{2}\right) \mathrm{d} x+\epsilon_{0} c_{N_{3}} \int_{0}^{L} \psi_{t}^{2} \mathrm{~d} x+\left(\frac{k_{2} \rho_{1}}{k_{1}}-\rho_{2}\right) \int_{0}^{L} \psi_{t} \varphi_{x t} \mathrm{~d} x \quad \text { if } i=2, \\
c_{N_{3}, \epsilon_{0}}^{L}\left(\varphi_{t}^{L}+\psi_{t}^{2}\right) \mathrm{d} x+\epsilon_{0} c_{N_{3}} \int_{0}^{L} w_{t}^{2} \mathrm{~d} x+\rho_{1}\left(\frac{k_{3}}{k_{1}}-1\right) \int_{0}^{L} w_{t} \varphi_{x t} \mathrm{~d} x \quad \text { if } i=3 .
\end{array}\right.
\end{aligned}
$$

At this point, we choose carefully the constants $N, N_{i}, \delta_{i}, \epsilon_{i}$ to get suitable values of $l_{i}$.

Case $i=1$. We choose

$$
\delta_{1}=\sqrt{\frac{k_{3}}{k_{0}+g_{3}^{0}}}, \quad \epsilon_{1}=\sqrt{\frac{\left(k_{1}+k_{3}\right) k_{3}}{\left(k_{2}-g_{2}^{0}\right) k_{1}}}
$$

(notice that $\epsilon_{1}$ is well defined thanks to (41)),

$$
N_{3}>\frac{l k_{3}}{2 k_{1}^{2} \epsilon_{1}} \text { and } \max \left\{k_{1} N_{3}-1,-k_{3} N_{3}+\frac{l\left(k_{2}-g_{2}^{0}\right) \epsilon_{1}}{2 k_{1}}, \frac{l k_{3}}{2 k_{1} \epsilon_{1}}\right\}<N_{4}<k_{1} N_{3} .
$$

We remark that $N_{4}$ exists according to the choice of $N_{3}$. By virtue of the choice of $\delta_{1}, \epsilon_{1}, N_{3}$ and $N_{4}$, we see that

$$
\max \left\{l_{1}, l_{4}, l_{5}, l_{6}\right\}<0 .
$$

On the other hand, from (33) (in case (1)), we find that

$$
g_{3}^{0} l_{8} \int_{0}^{L} w_{x}^{2} \mathrm{~d} x \leq \frac{g_{3}^{0} l_{8}}{k_{0}+g_{3}^{0}} \int_{0}^{L}\left(\left(k_{2}-g_{2}^{0}-k_{0}\right) \psi_{x}^{2}+k_{1}\left(\varphi_{x}+\psi+l w\right)^{2}+k_{3}\left(w_{x}-l \varphi\right)^{2}\right) \mathrm{d} x .
$$

Because $\tilde{l}_{6}=l_{7}=0$ and $l_{4}, l_{5}, \tilde{l}_{5}, l_{6}$ and $l_{8}$ do not depend on $g_{3}^{0}$, then, if $g_{3}^{0}$ is small enough so that

$$
\lambda_{0}:=\max \left\{l_{4}+\frac{\left(k_{2}-g_{2}^{0}-k_{0}\right) g_{3}^{0} l_{8}}{k_{0}+g_{3}^{0}}, l_{5}+g_{3}^{0} \tilde{l}_{5}+\frac{k_{3} g_{3}^{0} l_{8}}{k_{0}+g_{3}^{0}}, l_{6}+\frac{k_{1} g_{3}^{0} l_{8}}{k_{0}+g_{3}^{0}}\right\}<0,
$$


we get

$$
\begin{aligned}
& \int_{0}^{L}\left(l_{4} \psi_{x}^{2}+\left(l_{5}+g_{3}^{0} \tilde{l}_{5}\right)\left(w_{x}-l \varphi\right)^{2}+\left(l_{6}+g_{1}^{0} \tilde{l}_{6}\right)\left(\varphi_{x}+\psi+l w\right)^{2}+g_{1}^{0} l_{7} \varphi_{x}^{2}+g_{3}^{0} l_{8} w_{x}^{2}\right) \mathrm{d} x \\
& \leq \lambda_{0} \int_{0}^{L}\left(\psi_{x}^{2}+\left(w_{x}-l \varphi\right)^{2}+\left(\varphi_{x}+\psi+l w\right)^{2}\right) \mathrm{d} x .
\end{aligned}
$$

After, we choose $\epsilon_{0}>0$ small enough such that $\epsilon_{0} c_{N_{3}}+l_{1}<0$ and $N_{1}$ large enough so that

$$
\max \left\{l_{2}+c_{N_{3}, \epsilon_{0}}, l_{3}+c_{N_{3}, \epsilon_{0}}\right\}<0 .
$$

Finally, we choose $\delta_{0}>0$ small enough such that

$$
\tilde{c}_{1}:=-\max \left\{\frac{2}{\rho_{1}}\left(l_{1}+\epsilon_{0} c_{N_{3}}\right), \frac{2}{\rho_{2}}\left(l_{2}+c_{N_{3}, \epsilon_{0}}\right), \frac{2}{\rho_{1}}\left(l_{3}+c_{N_{3}, \epsilon_{0}}\right), \frac{2 \lambda_{0}}{k_{2}}, \frac{2 \lambda_{0}}{k_{3}}, \frac{2 \lambda_{0}}{k_{1}}\right\}-\delta_{0} c_{N_{1}, N_{3}, N_{4}}>0 .
$$

Then, using (43) and (70), we deduce from (110) that the estimate

$$
\begin{aligned}
F_{i}^{\prime}(t) \leq & -\tilde{c}_{1} E_{i}(t)+(N-c) E_{i}^{\prime}(t)+c \sum_{j \in\{1,2,3\} \backslash\{i\}} \int_{0}^{L} \int_{0}^{+\infty} g_{j}(s)\left(\partial_{x} \eta_{j}\right)^{2} \mathrm{~d} s \mathrm{~d} x \\
& +\left\{\begin{array}{l}
\left(\rho_{2}-\frac{k_{2} \rho_{1}}{k_{1}}\right) \int_{0}^{L} \psi_{t} \varphi_{x t} \mathrm{~d} x \text { if } i=1, \\
\left(\frac{k_{2} \rho_{1}}{k_{1}}-\rho_{2}\right) \int_{0}^{L} \psi_{t} \varphi_{x t} \mathrm{~d} x \text { if } i=2, \\
\rho_{1}\left(\frac{k_{3}}{k_{1}}-1\right) \int_{0}^{L} w_{t} \varphi_{x t} \mathrm{~d} x \text { if } i=3
\end{array}\right.
\end{aligned}
$$

is satisfied, for $i=1$.

Case $i=2$. As in the previous case, we choose

$$
\begin{aligned}
& \delta_{1}>\frac{\tilde{c}_{0}}{2}, \quad \epsilon_{2}=\frac{2 k_{1}}{l k_{2}}, \\
& \frac{\left(l k_{2} \epsilon_{2}-2 k_{1}\right) \delta_{1}}{2\left(k_{1}+k_{3}\right) \delta_{1}-\tilde{c}_{0} k_{3}}<N_{2}<\frac{2 k_{2}}{k_{1} \delta_{1}}\left(1-\frac{l k_{3}}{2 k_{1} \epsilon_{2}}\right), \quad \frac{l k_{2} \epsilon_{2}-2 k_{1}\left(N_{2}+1\right)}{2 k_{1} k_{3}}<N_{3}<\frac{\left(2 \delta_{1}-\tilde{c}_{0}\right) N_{2}}{2 k_{1} \delta_{1}}, \\
& \max \left\{\left(\frac{k_{1} \delta_{1}}{2 k_{2}}+1\right) N_{2}+\frac{l k_{3}}{2 k_{1} \epsilon_{2}},-k_{3} N_{3}+\frac{l k_{2} \epsilon_{2}}{2 k_{1}}, k_{1} N_{3}+\frac{\tilde{c}_{0} N_{2}}{2 \delta_{1}}+1\right\}<N_{4}<N_{2}+1, \\
& \epsilon_{1}, \delta_{2} \text { and } \delta_{3} \text { are any positive numbers. }
\end{aligned}
$$

By virtue of the choice of $\delta_{1}$ and $\epsilon_{2}$ and the hypothesis on $l$ in (61), we see that $N_{2}, N_{3}$ and $N_{4}$ exist and

$$
\max \left\{l_{2}, l_{4}, l_{5}, l_{6}\right\}<0 .
$$

On the other hand, from (33) (in case (2)), we find, similarly to (111),

$$
g_{1}^{0} l_{7} \int_{0}^{L} \varphi_{x}^{2} \mathrm{~d} x \leq \frac{g_{1}^{0} l_{7}}{k_{0}+g_{1}^{0}} \int_{0}^{L}\left(\left(k_{2}-k_{0}\right) \psi_{x}^{2}+k_{1}\left(\varphi_{x}+\psi+l w\right)^{2}+k_{3}\left(w_{x}-l \varphi\right)^{2}\right) \mathrm{d} x
$$


and

$$
g_{3}^{0} l_{8} \int_{0}^{L} w_{x}^{2} \mathrm{~d} x \leq \frac{g_{3}^{0} l_{8}}{k_{0}+g_{3}^{0}} \int_{0}^{L}\left(\left(k_{2}-k_{0}\right) \psi_{x}^{2}+k_{1}\left(\varphi_{x}+\psi+l w\right)^{2}+k_{3}\left(w_{x}-l \varphi\right)^{2}\right) \mathrm{d} x .
$$

Therefore, if $g_{1}^{0}$ and $g_{3}^{0}$ are small enough so that

$$
\begin{aligned}
& \lambda_{0}:=\max \left\{l_{4}+\left(k_{2}-k_{0}\right)\left(\frac{g_{1}^{0} l_{7}}{k_{0}+g_{1}^{0}}+\frac{g_{3}^{0} l_{8}}{k_{0}+g_{3}^{0}}\right), l_{5}+g_{3}^{0} \tilde{l}_{5}+k_{3}\left(\frac{g_{1}^{0} l_{7}}{k_{0}+g_{1}^{0}}+\frac{g_{3}^{0} l_{8}}{k_{0}+g_{3}^{0}}\right),\right. \\
& \left.l_{6}+g_{1}^{0} \tilde{l}_{6}+k_{1}\left(\frac{g_{1}^{0} l_{7}}{k_{0}+g_{1}^{0}}+\frac{g_{3}^{0} l_{8}}{k_{0}+g_{3}^{0}}\right)\right\}<0
\end{aligned}
$$

(notice that $l_{4}, l_{5}, \tilde{l}_{5}, l_{6}, \tilde{l}_{6}, l_{7}$ and $l_{8}$ do not depend neither on $g_{1}^{0}$ nor on $g_{3}^{0}$ ), then (113) holds. After, we choose $\epsilon_{0}>0$ small enough such that $\epsilon_{0} c_{N_{3}}+l_{2}<0$ and $N_{1}$ large enough so that

$$
\max \left\{l_{1}+c_{N_{3}, \epsilon_{0}}, l_{3}+c_{N_{3}, \epsilon_{0}}\right\}<0 .
$$

Finally, we choose $\delta_{0}>0$ small enough such that

$$
\tilde{c}_{1}:=-\max \left\{\frac{2}{\rho_{1}}\left(l_{1}+c_{N_{3}, \epsilon_{0}}\right), \frac{2}{\rho_{2}}\left(l_{2}+\epsilon_{0} c_{N_{3}}\right), \frac{2}{\rho_{1}}\left(l_{3}+c_{N_{3}, \epsilon_{0}}\right), \frac{2 \lambda_{0}}{k_{2}}, \frac{2 \lambda_{0}}{k_{3}}, \frac{2 \lambda_{0}}{k_{1}}\right\}-\delta_{0} c_{N_{1}, N_{3}, N_{4}}>0,
$$

and we deduce (114), for $i=2$, from (43), (70) and (110).

Case $i=3$. Similarly to the above two cases, we choose

$$
\max \left\{l-k_{1} N_{3}, k_{3} N_{3}-\frac{l k_{3}}{k_{1}}\right\}<N_{4}<k_{3} N_{3}+l,
$$

$N_{3}, \delta_{1}$ and $\epsilon_{1}$ are any positive numbers.

We have $\tilde{l}_{5}=l_{8}=0$ and thanks to the choice of $N_{4}$ we see that

$$
\max \left\{l_{3}, l_{4}, l_{5}, l_{6}\right\}<0 .
$$

On the other hand, similarly to (111), we get from (33) (in case (3)) that

$$
g_{1}^{0} l_{7} \int_{0}^{L} \varphi_{x}^{2} \mathrm{~d} x \leq \frac{g_{1}^{0} l_{7}}{k_{0}+g_{1}^{0}} \int_{0}^{L}\left(\left(k_{2}-g_{2}^{0}-k_{0}\right) \psi_{x}^{2}+k_{1}\left(\varphi_{x}+\psi+l w\right)^{2}+k_{3}\left(w_{x}-l \varphi\right)^{2}\right) \mathrm{d} x .
$$

Therefore, if $g_{1}^{0}$ is small enough so that

$$
\lambda_{0}:=\max \left\{l_{4}+\frac{g_{1}^{0} l_{7}\left(k_{2}-g_{2}^{0}-k_{0}\right)}{k_{0}+g_{1}^{0}}, l_{5}+\frac{k_{3} g_{1}^{0} l_{7}}{k_{0}+g_{1}^{0}}, l_{6}+g_{1}^{0} \tilde{l}_{6}+\frac{k_{1} g_{1}^{0} l_{7}}{k_{0}+g_{1}^{0}}\right\}<0,
$$

then (113) holds (the condition (119) holds, since $l_{4}, l_{5}, l_{6}, \tilde{l}_{6}$ and $l_{7}$ do not depend on $\left.g_{1}^{0}\right)$. After, we choose $\epsilon_{0}>0$ small enough such that $\epsilon_{0} c_{N_{3}}+l_{3}<0$ and $N_{1}$ large enough so that

$$
\max \left\{l_{1}+c_{N_{3}, \epsilon_{0}}, l_{2}+c_{N_{3}, \epsilon_{0}}\right\}<0 .
$$

Finally, we choose $\delta_{0}>0$ small enough such that

$$
\tilde{c}_{1}:=-\max \left\{\frac{2}{\rho_{1}}\left(l_{1}+c_{N_{3}, \epsilon_{0}}\right), \frac{2}{\rho_{2}}\left(l_{2}+c_{N_{3}, \epsilon_{0}}\right), \frac{2}{\rho_{1}}\left(l_{3}+\epsilon_{0} c_{N_{3}}\right), \frac{2 \lambda_{0}}{k_{2}}, \frac{2 \lambda_{0}}{k_{3}}, \frac{2 \lambda_{0}}{k_{1}}\right\}-\delta_{0} c_{N_{1}, N_{3}, N_{4}}>0,
$$

Then, (43), (70) and (110) give (114), for $i=3$.

Now, we estimate the integral of $g_{j}\left(\partial_{x} \eta_{j}\right)^{2}$ in (114), for $j \in\{1,2,3\} /\{i\}$. When (58) holds, we see that, by virtue of (71),

$$
\int_{0}^{L} \int_{0}^{+\infty} g_{j}(s)\left(\partial_{x} \eta_{j}\right)^{2} \mathrm{~d} s \mathrm{~d} x \leq-\frac{2}{\alpha} E_{i}^{\prime}(t)
$$


When (59) holds, we apply Lemma 3.6 [11] (in the particular case $B=-\partial_{x x}$ and $\|\cdot\|=\|\cdot\|_{L^{2}(] 0, L[)}$ ) to get the following inequality.

Lemma 4.6. There exists a positive constant $c$ such that, for any $\tau_{0}>0$, we have

$$
G^{\prime}\left(\tau_{0} E_{i}(t)\right) \int_{0}^{L} \int_{0}^{+\infty} g_{j}(s)\left(\partial_{x} \eta_{j}\right)^{2} d s d x \leq-c E_{i}^{\prime}(t)+c \tau_{0} E_{i}(t) G^{\prime}\left(\tau_{0} E_{i}(t)\right) .
$$

Proof. See Lemma 3.6 [11].

Using (120) and (121), we get in both cases (58) and (59)

$$
\begin{aligned}
& \frac{G_{0}\left(\tau_{0} E_{i}(t)\right)}{\tau_{0} E_{i}(t)} \sum_{j \in\{1,2,3\} \backslash\{i\}} \int_{0}^{L} \int_{0}^{+\infty} g_{j}(s)\left(\partial_{x} \eta_{j}\right)^{2} \mathrm{~d} s \mathrm{~d} x \leq c G_{0}\left(\tau_{0} E_{i}(t)\right)-c E_{i}^{\prime}(t) \\
& -c \frac{G_{0}\left(\tau_{0} E_{i}(t)\right)}{\tau_{0} E_{i}(t)} E_{i}^{\prime}(t),
\end{aligned}
$$

where $G_{0}$ is defined in (64). By multiplying (114) by $\frac{G_{0}\left(\tau_{0} E_{i}(t)\right)}{E_{i}(t)}$ and combining with (122), we obtain

$$
\begin{aligned}
& \frac{G_{0}\left(\tau_{0} E_{i}(t)\right)}{E_{i}(t)} F_{i}^{\prime}(t) \leq-\left(\tilde{c}_{1}-c \tau_{0}\right) G_{0}\left(\tau_{0} E_{i}(t)\right)+\left((N-c) \frac{G_{0}\left(\tau_{0} E_{i}(t)\right)}{E_{i}(t)}-c \tau_{0}\right) E_{i}^{\prime}(t) \\
& +\frac{G_{0}\left(\tau_{0} E_{i}(t)\right)}{E_{i}(t)}\left\{\begin{array}{l}
\left(\rho_{2}-\frac{k_{2} \rho_{1}}{k_{1}}\right) \int_{0}^{L} \psi_{t} \varphi_{x t} \mathrm{~d} x \text { if } i=1, \\
\left(\frac{k_{2} \rho_{1}}{k_{1}}-\rho_{2}\right) \int_{0}^{L} \psi_{t} \varphi_{x t} \mathrm{~d} x \text { if } i=2, \\
\rho_{1}\left(\frac{k_{3}}{k_{1}}-1\right) \int_{0}^{L} w_{t} \varphi_{x t} \mathrm{~d} x \text { if } i=3 .
\end{array}\right.
\end{aligned}
$$

On the other hand, from (33), (43) and (70), we deduce that there exists a positive constant $\gamma_{i}$ (not depending on $N$ ) satisfying

$$
\left|J_{i}+N_{2} D+N_{3} P+N_{4} R+N_{1} \sum_{j \in\{1,2,3\} \backslash\{i\}} I_{j}\right| \leq \gamma_{i} E_{i},
$$

which, combined with (108), implies that

$$
\left(N-\gamma_{i}\right) E_{i} \leq F_{i} \leq\left(N+\gamma_{i}\right) E_{i}
$$

Choosing $N$ so that

$$
N \geq c \quad \text { and } \quad N>\gamma_{i}
$$


( $c$ is the constant in (123)) and using (123), (124) and $E_{i}^{\prime} \leq 0$, we deduce that $F_{i} \sim E_{i}$ and

$$
\begin{aligned}
& \frac{G_{0}\left(\tau_{0} E_{i}(t)\right)}{E_{i}(t)} F_{i}^{\prime}(t) \leq-\left(\tilde{c}_{1}-c \tau_{0}\right) G_{0}\left(\tau_{0} E_{i}(t)\right)-c \tau_{0} E_{i}^{\prime}(t) \\
& +\frac{G_{0}\left(\tau_{0} E_{i}(t)\right)}{E_{i}(t)}\left\{\begin{array}{l}
\left(\rho_{2}-\frac{k_{2} \rho_{1}}{k_{1}}\right) \int_{0}^{L} \psi_{t} \varphi_{x t} \mathrm{~d} x \text { if } i=1, \\
\left(\frac{k_{2} \rho_{1}}{k_{1}}-\rho_{2}\right) \int_{0}^{L} \psi_{t} \varphi_{x t} \mathrm{~d} x \text { if } i=2, \\
\rho_{1}\left(\frac{k_{3}}{k_{1}}-1\right) \int_{0}^{L} w_{t} \varphi_{x t} \mathrm{~d} x \text { if } i=3 .
\end{array}\right.
\end{aligned}
$$

Let $\tilde{\tau}_{i}>0$ and

$$
\tilde{F}_{i}=\tilde{\tau}_{i}\left(\frac{G_{0}\left(\tau_{0} E_{i}(t)\right)}{E_{i}(t)} F_{i}+c \tau_{0} E_{i}(t)\right)
$$

Because $\frac{G_{0}\left(\tau_{0} E_{i}(t)\right)}{E_{i}(t)}$ is nonincreasing, then, thanks to (124),

$$
c \tilde{\tau}_{i} \tau_{0} E_{i} \leq \tilde{F}_{i} \leq \tilde{\tau}_{i}\left(\left(N+\gamma_{i}\right) \frac{G_{0}\left(\tau_{0} E_{i}(0)\right)}{E_{i}(0)}+c \tau_{0}\right) E_{i}
$$

Let us choose $\tilde{\tau}_{i}>0$ such that

$$
\tilde{F}_{i} \leq \tau_{0} E_{i} \quad \text { and } \quad \tilde{F}_{i}(0) \leq 1 .
$$

We have, using (125), (126) and the fact that $\frac{G_{0}\left(\tau_{0} E_{i}\right)}{E_{i}}$ is nonincreasing,

$$
\begin{aligned}
\tilde{F}_{i}^{\prime}(t) \leq- & \tilde{\tau}_{i}\left(\tilde{c}_{1}-c \tau_{0}\right) G_{0}\left(\tau_{0} E_{i}(t)\right) \\
+ & \left(\tilde { \tau } _ { i } \frac { G _ { 0 } ( \tau _ { 0 } E _ { i } ( t ) ) } { E _ { i } ( t ) } \left\{\begin{array}{l}
\left(\frac{k_{2} \rho_{1}}{k_{1}}\right) \int_{0}^{L} \psi_{t} \varphi_{x t} \mathrm{~d} x \text { if } i=1, \\
\left(\frac{k_{2} \rho_{1}}{k_{1}}-\rho_{2}\right) \int_{0}^{L} \psi_{t} \varphi_{x t} \mathrm{~d} x \text { if } i=2, \\
\rho_{1}\left(\frac{k_{3}}{k_{1}}-1\right) \int_{0}^{L} w_{t} \varphi_{x t} \mathrm{~d} x \text { if } i=3 .
\end{array}\right.\right.
\end{aligned}
$$

According to (60), the coefficients of the integrals in (129) vanish, and hence, by choosing $\tau_{0}>0$ small enough such that $\tilde{c}_{1}-c \tau_{0}>0$ and using the first inequality in (128), we get, for $c_{i}^{\prime}=\tilde{\tau}_{i}\left(\tilde{c}_{1}-c \tau_{0}\right)$,

$$
\tilde{F}_{i}^{\prime} \leq-c_{i}^{\prime} G_{0}\left(\tilde{F}_{i}\right),
$$

whereupon

$$
\left(\tilde{G}\left(\tilde{F}_{i}\right)\right)^{\prime} \geq c_{i}^{\prime},
$$

where $\tilde{G}(t)=\int_{t}^{1} \frac{1}{G_{0}(s)} \mathrm{d} s$. Integrating (131) over $[0, t]$ yields

$$
\tilde{G}\left(\tilde{F}_{i}(t)\right) \geq c_{i}^{\prime} t+\tilde{G}\left(\tilde{F}_{i}(0)\right)
$$


Because $\tilde{F}_{i}(0) \leq 1$ (from (128)), $\tilde{G}(1)=0$ and $\tilde{G}$ is decreasing, we obtain from (132) that

$$
\tilde{G}\left(\tilde{F}_{i}(t)\right) \geq c_{i}^{\prime} t
$$

which implies that

$$
\tilde{F}_{i}(t) \leq \tilde{G}^{-1}\left(c_{i}^{\prime} t\right)
$$

Then, (70) and (127) give (63).

\section{Proof of weak decay $(66)$}

In this section, we treat the case when (60) does not hold which is more realistic from the physical point of view. We need to estimate the integrals in (125) using the following systems resulting from differentiating, respectively, (1)-(3) with respect to time $t$ :

$$
\begin{aligned}
& \left\{\begin{array}{l}
\rho_{1} \varphi_{t t t}-k_{1}\left(\varphi_{x t}+\psi_{t}+l w_{t}\right)_{x}-l k_{3}\left(w_{x t}-l \varphi_{t}\right)=0 \\
\rho_{2} \psi_{t t t}-k_{2} \psi_{x x t}+k_{1}\left(\varphi_{x t}+\psi_{t}+l w_{t}\right)+\int_{0}^{+\infty} g_{2}(s) \psi_{x x t}(x, t-s) \mathrm{d} s=0, \\
\rho_{1} w_{t t t}-k_{3}\left(w_{x t}-l \varphi_{t}\right)_{x}+l k_{1}\left(\varphi_{x t}+\psi_{t}+l w_{t}\right)+\int_{0}^{+\infty} g_{3}(s) w_{x x t}(x, t-s) \mathrm{d} s=0, \\
\varphi_{t}(0, t)=\psi_{x t}(0, t)=w_{x t}(0, t)=\varphi_{t}(L, t)=\psi_{x t}(L, t)=w_{x t}(L, t)=0, \\
\rho_{1} \varphi_{t t t}-k_{1}\left(\varphi_{x t}+\psi_{t}+l w_{t}\right)_{x}-l k_{3}\left(w_{x t}-l \varphi_{t}\right)+\int_{0}^{+\infty} g_{1}(s) \varphi_{x x t}(x, t-s) \mathrm{d} s=0, \\
\rho_{2} \psi_{t t t}-k_{2} \psi_{x x t}+k_{1}\left(\varphi_{x t}+\psi_{t}+l w_{t}\right)=0, \quad{ }_{0}^{+\infty} \\
\rho_{1} w_{t t t}-k_{3}\left(w_{x t}-l \varphi_{t}\right)_{x}+l k_{1}\left(\varphi_{x t}+\psi_{t}+l w_{t}\right)+\int_{0}^{+\infty} g_{3}(s) w_{x x t}(x, t-s) \mathrm{d} s=0, \\
\varphi_{t}(0, t)=\psi_{x t}(0, t)=w_{x t}(0, t)=\varphi_{t}(L, t)=\psi_{x t}(L, t)=w_{x t}(L, t)=0
\end{array}\right.
\end{aligned}
$$

and

$$
\left\{\begin{array}{l}
\rho_{1} \varphi_{t t t}-k_{1}\left(\varphi_{x t}+\psi_{t}+l w_{t}\right)_{x}-l k_{3}\left(w_{x t}-l \varphi_{t}\right)+\int_{0}^{+\infty} g_{1}(s) \varphi_{x x t}(x, t-s) \mathrm{d} s=0 \\
\rho_{2} \psi_{t t t}-k_{2} \psi_{x x t}+k_{1}\left(\varphi_{x t}+\psi_{t}+l w_{t}\right)+\int_{0}^{+\infty} g_{2}(s) \psi_{x x t}(x, t-s) \mathrm{d} s=0 \\
\rho_{1} w_{t t t}-k_{3}\left(w_{x t}-l \varphi_{t}\right)_{x}+l k_{1}\left(\varphi_{x t}+\psi_{t}+l w_{t}\right)=0 \\
\varphi_{t}(0, t)=\psi_{x t}(0, t)=w_{x t}(0, t)=\varphi_{t}(L, t)=\psi_{x t}(L, t)=w_{x t}(L, t)=0
\end{array}\right.
$$

Systems (133)-(135) are well posed for initial data $U_{i}^{0} \in D\left(\mathcal{A}_{i}^{n}\right), i=1,2,3$, respectively, and $n \in \mathbb{N}^{*}$ thanks to Theorem 2.3, where

$$
\partial_{t} U_{i} \in \cap_{k=0}^{n-1} C^{n-1-k}\left(\mathbb{R}_{+} ; D\left(\mathcal{A}_{i}^{k}\right)\right) .
$$


Let $U_{i}^{0} \in D\left(\mathcal{A}_{i}\right)$ and $\tilde{E}_{i}, i=1,2,3$, be the energy of (133)-(135), respectively, defined by

$$
\tilde{E}_{i}(t)=\frac{1}{2}\left\|\partial_{t} U_{i}(t)\right\|_{\mathcal{H}_{i}}^{2} .
$$

As for (71), we have

$$
\tilde{E}_{i}^{\prime}(t)=\frac{1}{2} \begin{cases}\int_{0}^{L} \int_{0}^{+\infty}\left(g_{2}^{\prime}\left(\partial_{x t} \eta_{2}\right)^{2}+g_{3}^{\prime}\left(\partial_{x t} \eta_{3}\right)^{2}\right) \mathrm{d} s \mathrm{~d} x & \text { if } i=1, \\ \int_{0}^{L} \int_{0}^{+\infty}\left(g_{1}^{\prime}\left(\partial_{x t} \eta_{1}\right)^{2}+g_{3}^{\prime}\left(\partial_{x t} \eta_{3}\right)^{2}\right) \mathrm{d} s \mathrm{~d} x & \text { if } i=2, \\ \int_{0}^{L} \int_{0}^{+\infty}\left(g_{1}^{\prime}\left(\partial_{x t} \eta_{1}\right)^{2}+g_{2}^{\prime}\left(\partial_{x t} \eta_{2}\right)^{2}\right) \mathrm{d} s \mathrm{~d} x & \text { if } i=3,\end{cases}
$$

so $\tilde{E}_{i}$ is nonincreasing. Let $\tilde{\tau}_{i}=1$ in (126). Then, (125) leads to

$$
\begin{aligned}
& \tilde{F}_{i}^{\prime}(t) \leq-\left(\tilde{c}_{1}-c \tau_{0}\right) G_{0}\left(\tau_{0} E_{i}(t)\right)\left(\rho_{2}-\frac{k_{2} \rho_{1}}{k_{1}}\right) \int_{0}^{L} \psi_{t} \varphi_{x t} \mathrm{~d} x \text { if } i=1, \\
&+\frac{G_{0}\left(\tau_{0} E_{i}(t)\right)}{E_{i}(t)}\left\{\begin{array}{l}
\left(\frac{k_{2} \rho_{1}}{k_{1}}-\rho_{2}\right) \int_{0}^{L} \psi_{t} \varphi_{x t} \mathrm{~d} x \text { if } i=2, \\
\rho_{1}\left(\frac{k_{3}}{k_{1}}-1\right) \int_{0}^{L} w_{t} \varphi_{x t} \mathrm{~d} x \text { if } i=3 .
\end{array}\right.
\end{aligned}
$$

We use an idea introduced in [9] to get the following lemma.

Lemma 5.1. For any $\epsilon>0$, (1)-(3) imply that,

$$
\begin{aligned}
\mid \begin{aligned}
\left(\rho_{2}-\frac{k_{2} \rho_{1}}{k_{1}}\right) \frac{G_{0}\left(\tau_{0} E_{1}(t)\right)}{E_{1}(t)} \int_{0}^{L} \psi_{t} \varphi_{x t} d x \mid \leq & c_{\epsilon} \frac{G_{0}\left(\tau_{0} E_{1}(t)\right)}{E_{1}(t)} \int_{0}^{L} \int_{0}^{+\infty} g_{2}(s)\left(\partial_{x t} \eta_{2}\right)^{2} d s d x \\
& +\epsilon G_{0}\left(\tau_{0} E_{1}(t)\right)-c_{\epsilon} \frac{G_{0}\left(\tau_{0} E_{1}(0)\right)}{E_{1}(0)} E_{1}^{\prime}(t),
\end{aligned} \\
\mid \begin{aligned}
\left(\frac{k_{2} \rho_{1}}{k_{1}}-\rho_{2}\right) \frac{G_{0}\left(\tau_{0} E_{2}(t)\right)}{E_{2}(t)} \int_{0}^{L} \psi_{t} \varphi_{x t} d x \mid \leq & c_{\epsilon} \frac{G_{0}\left(\tau_{0} E_{2}(t)\right)}{E_{2}(t)} \int_{0}^{L} \int_{0}^{+\infty} g_{1}(s)\left(\partial_{x t} \eta_{1}\right)^{2} d s d x \\
& +\epsilon G_{0}\left(\tau_{0} E_{2}(t)\right)-c_{\epsilon} \frac{G_{0}\left(\tau_{0} E_{2}(0)\right)}{E_{2}(0)} E_{2}^{\prime}(t)
\end{aligned}
\end{aligned}
$$

and

$$
\begin{aligned}
& \left|\rho_{1}\left(\frac{k_{3}}{k_{1}}-1\right) \frac{G_{0}\left(\tau_{0} E_{3}(t)\right)}{E_{3}(t)} \int_{0}^{L} w_{t} \varphi_{x t} d x\right| \leq c_{\epsilon} \frac{G_{0}\left(\tau_{0} E_{3}(t)\right)}{E_{3}(t)} \int_{0}^{L} \int_{0}^{+\infty} g_{1}(s)\left(\partial_{x t} \eta_{1}\right)^{2} d s d x \\
& +\epsilon G_{0}\left(\tau_{0} E_{3}(t)\right)-c_{\epsilon} \frac{G_{0}\left(\tau_{0} E_{3}(0)\right)}{E_{3}(0)} E_{3}^{\prime}(t) .
\end{aligned}
$$


Proof. We have, by integrating with respect to $x$ and using the definition of $\eta_{2}$,

$$
\begin{aligned}
\left(\rho_{2}-\frac{k_{2} \rho_{1}}{k_{1}}\right) \int_{0}^{L} \psi_{t} \varphi_{x t} \mathrm{~d} x= & -\left(\rho_{2}-\frac{k_{2} \rho_{1}}{k_{1}}\right) \int_{0}^{L} \varphi_{t} \psi_{x t} \mathrm{~d} x \\
= & -\frac{1}{g_{2}^{0}}\left(\rho_{2}-\frac{k_{2} \rho_{1}}{k_{1}}\right) \int_{0}^{L} \varphi_{t} \int_{0}^{+\infty} g_{2}(s) \partial_{x t} \eta_{2} \mathrm{~d} s \mathrm{~d} x \\
& -\frac{1}{g_{2}^{0}}\left(\rho_{2}-\frac{k_{2} \rho_{1}}{k_{1}}\right) \int_{0}^{L} \varphi_{t} \int_{0}^{+\infty} g_{2}(s) \psi_{x t}(t-s) \mathrm{d} s \mathrm{~d} x .
\end{aligned}
$$

Using (81) (for $\eta=\partial_{x t} \eta_{2}$ and $v=\varphi_{t}$ ) and (70) (for $i=1$ ), we get, for all $\epsilon>0$,

$$
\begin{aligned}
\left|\frac{1}{g_{2}^{0}}\left(\rho_{2}-\frac{k_{2} \rho_{1}}{k_{1}}\right) \int_{0}^{L} \varphi_{t} \int_{0}^{+\infty} g_{2}(s) \partial_{x t} \eta_{2} \mathrm{~d} s \mathrm{~d} x\right| \leq & \frac{\epsilon}{2} E_{1}(t) \\
& +c_{\epsilon} \int_{0}^{L} \int_{0}^{+\infty} g_{2}(s)\left(\partial_{x t} \eta_{2}\right)^{2} \mathrm{~d} s \mathrm{~d} x .
\end{aligned}
$$

On the other hand, by integrating with respect to $s$ and using the definition of $\eta_{2}$, we obtain

$$
\begin{aligned}
\int_{0}^{L} \varphi_{t} \int_{0}^{+\infty} g_{2}(s) \psi_{x t}(t-s) \mathrm{d} s \mathrm{~d} x & =-\int_{0}^{L} \varphi_{t} \int_{0}^{+\infty} g_{2}(s) \partial_{s}\left(\psi_{x}(t-s)\right) \mathrm{d} s \mathrm{~d} x \\
& =\int_{0}^{L} \varphi_{t}\left(g_{2}(0) \psi_{x}(t)+\int_{0}^{+\infty} g_{2}^{\prime}(s) \psi_{x}(t-s) \mathrm{d} s\right) \mathrm{d} x \\
& =-\int_{0}^{L} \varphi_{t} \int_{0}^{+\infty} g_{2}^{\prime}(s) \partial_{x} \eta_{2} \mathrm{~d} s \mathrm{~d} x .
\end{aligned}
$$

Therefore, using (82) (for $\eta=\partial_{x} \eta_{2}$ and $v=\varphi_{t}$ ) and (71) (for $i=1$ ),

$$
\left|\frac{1}{g_{2}^{0}}\left(\rho_{2}-\frac{k_{2} \rho_{1}}{k_{1}}\right) \int_{0}^{L} \varphi_{t} \int_{0}^{+\infty} g_{2}(s) \psi_{x t}(t-s) \mathrm{d} s \mathrm{~d} x\right| \leq \frac{\epsilon}{2} E_{1}(t)-c_{\epsilon} E_{1}^{\prime}(t) .
$$

Inserting (143) and (144) into (142), multiplying by $\frac{G_{0}\left(\tau_{0} E_{1}\right)}{E_{1}}$ and noting that $\frac{G_{0}\left(\tau_{0} E_{1}\right)}{E_{1}}$ is nonincreasing, we obtain (139). Using the same arguments, we get (140) and (141). 
Now, by combining (138)-(141), and choosing $\epsilon=\frac{\tilde{c}_{1}}{2}$, we get

$$
\begin{aligned}
\tilde{F}_{i}^{\prime}(t) \leq & -\left(\frac{\tilde{c}_{1}}{2}-c \tau_{0}\right) G_{0}\left(\tau_{0} E_{i}(t)\right)-c \frac{G_{0}\left(\tau_{0} E_{i}(0)\right)}{E_{i}(0)} E_{i}^{\prime}(t) \\
& +c \frac{G_{0}\left(\tau_{0} E_{i}(t)\right)}{E_{i}(t)}\left\{\begin{array}{l}
\int_{0}^{L} \int_{0}^{+\infty} g_{2}(s)\left(\partial_{x t} \eta_{2}\right)^{2} \mathrm{~d} s \mathrm{~d} x \text { if } i=1, \\
\int_{0}^{L} \int_{0}^{+\infty} g_{1}(s)\left(\partial_{x t} \eta_{1}\right)^{2} \mathrm{~d} s \mathrm{~d} x \text { if } i=2 \text { or } i=3 .
\end{array}\right.
\end{aligned}
$$

Similarly to (120) and (121), using (137), we find, for $j \in\{1,2,3\} \backslash\{i\}$,

$$
\int_{0}^{L} \int_{0}^{+\infty} g_{j}(s)\left(\partial_{x t} \eta_{j}\right)^{2} \mathrm{~d} s \mathrm{~d} x \leq-\frac{2}{\alpha} \tilde{E}_{i}^{\prime}(t)
$$

when (58) holds. When (59) holds, there exists a positive constant $c$ such that, for any $\tau_{0}>0$, we have as for (121) (see the proof of Lemma 3.6 [11])

$$
G^{\prime}\left(\tau_{0} E_{i}(t)\right) \int_{0}^{L} \int_{0}^{+\infty} g_{j}(s)\left(\partial_{x t} \eta_{j}\right)^{2} \mathrm{~d} s \mathrm{~d} x \leq-c \tilde{E}_{i}^{\prime}(t)+c \tau_{0} E_{i}(t) G^{\prime}\left(\tau_{0} E_{i}(t)\right) .
$$

From (146) and (147), we find that, in both cases (58) and (59),

$$
\begin{array}{r}
\frac{G_{0}\left(\tau_{0} E_{i}(t)\right)}{E_{i}(t)} \int_{0}^{L} \int_{0}^{+\infty} g_{j}(s)\left(\partial_{x t} \eta_{j}\right)^{2} \mathrm{~d} s \mathrm{~d} x \leq c \tau_{0} G_{0}\left(\tau_{0} E_{i}(t)\right)-c \frac{G_{0}\left(\tau_{0} E_{i}(t)\right)}{E_{i}(t)} \tilde{E}_{i}^{\prime}(t) \\
-c \tau_{0} \tilde{E}_{i}^{\prime}(t), \forall j \in\{1,2,3\} \backslash\{i\} .
\end{array}
$$

Inserting (148) in (145), choosing $\tau_{0}>0$ small enough such that $\frac{\tilde{c}_{1}}{2}-c \tau_{0}>0$ and using the fact that $\frac{G_{0}\left(\tau_{0} E_{i}\right)}{E_{i}}$ is nonincreasing, we find, for some $\tilde{c}_{2}>0$,

$$
G_{0}\left(\tau_{0} E_{i}(t)\right) \leq-\tilde{c}_{2} \tilde{F}_{i}^{\prime}(t)-c\left(1+\frac{G_{0}\left(\tau_{0} E_{i}(0)\right)}{E_{i}(0)}\right)\left(E_{i}^{\prime}(t)+\tilde{E}_{i}^{\prime}(t)\right) .
$$

By integration with respect to $t$ and using (127), we get, for some $\tilde{c}_{3}>0$,

$$
\int_{S}^{T} G_{0}\left(\tau_{0} E_{i}(t)\right) \mathrm{d} t \leq \tilde{c}_{3}\left(1+\frac{G_{0}\left(\tau_{0} E_{i}(0)\right)}{E_{i}(0)}\right)\left(E_{i}(S)+\tilde{E}_{i}(S)\right), \quad \forall T \geq S \geq 0 .
$$

Choosing $S=0$ in (150) and using the fact that $G_{0}\left(\tau_{0} E_{i}\right)$ is nonincreasing, we obtain

$$
G_{0}\left(\tau_{0} E_{i}(T)\right) T \leq \int_{0}^{T} G_{0}\left(E_{i}(t)\right) \mathrm{d} t \leq \tilde{c}_{3}\left(1+\frac{G_{0}\left(\tau_{0} E_{i}(0)\right)}{E_{i}(0)}\right)\left(E_{i}(0)+\tilde{E}_{i}(0)\right) .
$$

Because $G_{0}^{-1}$ is increasing, (66) for $n=1$ is deduced from (70) and (151) with

$$
c_{i, 1}=\max \left\{\frac{2}{\tau_{0}}, \tilde{c}_{3}\left(1+\frac{G_{0}\left(\tau_{0} E_{i}(0)\right)}{E_{i}(0)}\right)\left(E_{i}(0)+\tilde{E}_{i}(0)\right)\right\} .
$$

By induction on $n$, suppose that (66) holds and let $U_{i}^{0} \in D\left(\mathcal{A}_{i}^{n+1}\right)$ be such that (65) holds, for $n+1$ instead of $n$. We have $\partial_{t} U_{i}(0) \in D\left(\mathcal{A}_{i}^{n}\right)$ (thanks to Theorem 2.3) and $\partial_{t} U_{i}$ satisfies the first three equations 
and the boundary conditions of system $(i), i=1,2,3$ (that is, (133)-(135) are satisfied). On the other hand, let $\tilde{\eta}_{i}=\partial_{t} \eta_{i}, i=1,2,3$. From (11), we remark that

$$
\tilde{\eta}_{i}(x, t, s)= \begin{cases}\varphi_{t}(x, t)+\partial_{s}(\varphi(x, t-s)) & \text { if } i=1 \\ \psi_{t}(x, t)+\partial_{s}(\psi(x, t-s)) & \text { if } i=2, \\ w_{t}(x, t)+\partial_{s}(w(x, t-s)) & \text { if } i=3 .\end{cases}
$$

Then,

$$
\tilde{\eta}_{i}^{0}(x, s):=\tilde{\eta}_{i}(x, 0, s)= \begin{cases}\varphi_{1}(x)+\partial_{s} \varphi_{0}(x, s) & \text { if } i=1, \\ \psi_{1}(x)+\partial_{s} \psi_{0}(x, s) & \text { if } i=2, \\ w_{1}(x)+\partial_{s} w_{0}(x, s) & \text { if } i=3,\end{cases}
$$

therefore, for $k=1, \ldots, n$,

$$
\partial_{s}^{k} \tilde{\eta}_{i}^{0}(x, s)= \begin{cases}\partial_{s}^{k+1} \varphi_{0}(x, s) & \text { if } i=1 \\ \partial_{s}^{k+1} \psi_{0}(x, s) & \text { if } i=2 \\ \partial_{s}^{k+1} w_{0}(x, s) & \text { if } i=3\end{cases}
$$

But, again from (11),

$$
\partial_{s}^{k+1} \eta_{i}^{0}(x, s)=\partial_{s}^{k+1} \eta_{i}(x, 0, s)= \begin{cases}-\partial_{s}^{k+1} \varphi_{0}(x, s) & \text { if } i=1 \\ -\partial_{s}^{k+1} \psi_{0}(x, s) & \text { if } i=2 \\ -\partial_{s}^{k+1} w_{0}(x, s) & \text { if } i=3\end{cases}
$$

Then,

$$
\partial_{s}^{k} \tilde{\eta}_{i}^{0}(x, s)=-\partial_{s}^{k+1} \eta_{i}^{0}(x, s)
$$

Consequently, (65) is satisfied, for $\tilde{\eta}_{j}^{0}$ instead of $\eta_{j}^{0}, j \in\{1,2,3\} \backslash\{i\}$ (because (65) is supposed being satisfied, for $n+1$ instead of $n$ ). Hence, for some positive constant $\tilde{c}_{i, n}$,

$$
\left\|\partial_{t} U_{i}(t)\right\|_{\mathcal{H}_{i}}^{2} \leq \tilde{c}_{i, n} G_{n}\left(\frac{\tilde{c}_{i, n}}{t}\right), \quad \forall t>0
$$

Choosing $S=\frac{T}{2}$ in (150) and combining with (66) and (152), we conclude that $\left(G_{0}\left(\tau_{0} E_{i}\right)\right.$ is nonincreasing)

$$
G_{0}\left(\tau_{0} E_{i}(T)\right) T \leq 2 \int_{\frac{T}{2}}^{T} G_{0}\left(\tau_{0} E_{i}(t)\right) \mathrm{d} t \leq \tilde{c}_{3}\left(1+\frac{G_{0}\left(\tau_{0} E_{i}(0)\right)}{E_{i}(0)}\right)\left(c_{i, n} G_{n}\left(\frac{2 c_{i, n}}{T}\right)+\tilde{c}_{i, n} G_{n}\left(\frac{2 \tilde{c}_{i, n}}{T}\right)\right),
$$

which implies that, for

$$
c_{i, n+1}=\max \left\{\frac{2}{\tau_{0}}, \tilde{c}_{3}\left(c_{i, n}+\tilde{c}_{i, n}\right)\left(1+\frac{G_{0}\left(\tau_{0} E_{i}(0)\right)}{E_{i}(0)}\right), 2 c_{i, n}, 2 \tilde{c}_{i, n}\right\}
$$

and any $T>0$ (notice that $G_{n}$ is increasing),

$$
\begin{aligned}
& \left\|U_{i}(t)\right\|_{\mathcal{H}_{i}}^{2}=2 E_{i}(T) \leq c_{i, n+1} G_{0}^{-1}\left(\frac{c_{i, n+1}}{T} G_{n}\left(\frac{c_{i, n+1}}{T}\right)\right)=c_{i, n+1} G_{1}\left(\frac{c_{n+1}}{T} G_{n}\left(\frac{c_{n+1}}{T}\right)\right) \\
& \quad=c_{i, n+1} G_{n+1}\left(\frac{c_{i, n+1}}{T}\right),
\end{aligned}
$$

so (66) holds, for $n+1$. 
Remark 5.2. 1. It is possible to prove some similar results under other kinds of boundary conditions like the homogeneous Dirichlet-Dirichlet-Dirichlet ones.

2. One of the interesting questions related to our results is the optimality of the decay estimates (63) and (66). In case of linear frictional dampings or infinite memories with kernels satisfying (58), the optimality of (69), when $n=1$ and (7) does not hold, was proved in [2] and [6].

3. Another interesting question concerns the stability of Bresse systems with only one infinite memory and a kernel having a general growth at infinity (not necessarily of exponential type). The case (8) has been treated in [6] but for kernels $g$ satisfying (9), which implies (10); that is, $g$ converges exponentially to zero.

4. Our stability results (63) and (66) hold under the smallness conditions (61) on $l, g_{1}^{0}$ and $g_{3}^{0}$, and the boundedness conditions $(62)$ and $(65)$ on the initial data $\eta_{j}^{0}$. It is interesting to drop these purely technical conditions (61), (62) and (65).

\section{Acknowlegments}

The second-named author thanks the NNAM group, King Abdulaziz University and Jeddah University for their support.

\section{References}

1. Alabau-Boussouira, F., Muñoz Rivera, J.E., Almeida Júnior, D.S.: Stability to weak dissipative Bresse system. J. Math. Anal. Appl. 374, 481-498 (2011)

2. Alves, M.O., Fatori, L.H., Jorge Silva, M.A., Monteiro, R.N.: Stability and optimality of decay rate for weakly dissipative Bresse system. Math. Meth. Appl. Sci. 38, 898-908 (2015)

3. Bresse, J.A.C.: Cours de Méchanique Appliquée. Mallet Bachelier, Paris (1859)

4. Charles, W., Soriano, J.A., Nascimentoc, F.A., Rodrigues, J.H.: Decay rates for Bresse system with arbitrary nonlinear localized damping. J. Diff. Equa. 255, 2267-2290 (2013)

5. Dafermos, C.M.: Asymptotic stability in viscoelasticity. Arch. Rational Mech. Anal. 37, 297-308 (1970)

6. De Lima Santos, M., Soufyane, A., Da Silva Almeida Júnior, D.: Asymptotic behavior to Bresse system with past history. Quart. Appl. Math. 73, 23-54 (2015)

7. Fatori, L.H., Monteiro, R.N.: The optimal decay rate for a weak dissipative Bresse system. Appl. Math. Lett. 25, 600604 (2012)

8. Fatori, L.H., Muñoz Rivera, J.E.: Rates of decay to weak thermoelastic Bresse system. IMA J. Appl. Math. 75, 881904 (2010)

9. Fernández Sare, H.D., Muñoz Rivera, J.E.: Stability of Timoshenko systems with past history. J. Math. Anal. Appl. 339, 482-502 (2008)

10. Guesmia, A.: Asymptotic stability of abstract dissipative systems with infinite memory. J. Math. Anal. Appl. 382, 748$760(2011)$

11. Guesmia, A.: Asymptotic behavior for coupled abstract evolution equations with one infinite memory. Applicable Analysis 94, 184-217 (2015)

12. Guesmia, A., Kafini, M.: Bresse system with infinite memories. Math. Meth. Appl. Sci. 38, $2389-2402$ (2015)

13. Komornik, V.: Exact Controllability and Stabilization. The Multiplier Method. Masson-John Wiley, Paris (1994)

14. Lagnese, J.E., Leugering, G., Schmidt, J.P.: Modelling of dynamic networks of thin thermoelastic beams. Math. Meth. Appl. Scie. 16, 327-358 (1993)

15. Lagnese, J.E., Leugering, G., Schmidt, E.J.P.G.: Modelling, Analysis and Control of Dynamic Elastic Multi-Link Structures. Systems \& Control: Foundations \& Applications. Birkhäuser, Boston. ISBN: 0-8176-3705-2 (1994)

16. Liu, Z., Rao, B.: Energy decay rate of the thermoelastic Bresse system. Z. Angew. Math. Phys. 60, 54-69 (2009)

17. Najdi, N., Wehbe, A.: Weakly locally thermal stabilization of Bresse systems. Elec. J. Diff. Equa. 2014, 1-19 (2014)

18. Noun, N., Wehbe, A.: Weakly locally internal stabilization of elastic Bresse system. C. R. Acad. Scie. Paris Sér. I 350, 493498 (2012)

19. Pazy, A.: Semigroups of linear operators and applications to partial differential equations. Springer, New York (1983)

20. Soriano, J.A., Charles, W., Schulz, R.: Asymptotic stability for Bresse systems. J. Math. Anal. Appl. 412, 369-380 (2014) 
21. Soriano, J.A., Muñoz Rivera, J.E., Fatori, L.H.: Bresse system with indefinite damping. J. Math. Anal. Appl. 387, 284290 (2012)

22. Soufyane, A., Said-Houari, B.: The effect of the wave speeds and the frictional damping terms on the decay rate of the Bresse system. Evol. Equa. Cont. Theory 3, 713-738 (2014)

23. Wehbe, A., Youssef, W.: Exponential and polynomial stability of an elastic Bresse system with two locally distributed feedbacks. J. Math. Phys. 51, 1-17 (2010)

24. Youssef, W.: Contrôle et stabilisation de systèmes élastiques couplés, PhD thesis, University of Metz, France (2009)

A. Guesmia

Institut Elie Cartan de Lorraine

UMR 7502, Université de Lorraine

Bat. A, Ile du Saulcy

57045 Metz Cedex 01

France

e-mail: aissa.guesmia@univ-lorraine.fr

M. Kirane

LaSIE, Faculté des Sciences

Pôle Sciences et Technologies

Université de la Rochelle

La Rochelle

France

e-mail: mokhtar.kirane@univ-lr.fr

(Received: February 1, 2016; revised: July 6, 2016) 